\title{
The Polyakov Path Integral over Bordered Surfaces (The Open String Amplitudes)
}

\author{
Zbigniew Jaskólski \\ Institute of Theoretical Physics, University of Wroclaw, ul. Cybulskiego 36, \\ PL-50205 Wrocław, Poland
}

\begin{abstract}
We use the Feynman functional quantization scheme adapted to the gauge theories with reparametrization invariance to the functional covariant first quantization of the open bosonic BDHP string in a position representation. The consistent functional integral representation of the open string propagator is derived and evaluated. This result is used as a starting point for two kinds of constructions of the off-shell multiloop open string amplitudes. The general idea of the presented approach is to consider the off-shell amplitudes as functionals on the space $\mathscr{C}$ of contours endowed with an intrinsic metric or on the space $\mathscr{C} / \mathbb{R}_{+}$.
\end{abstract}

\section{Introduction}

The Polyakov path integral [1] over bordered surfaces was first considered in the context of dual models beyond the critical dimensions [2]. At the same time its application to the phenomenological model for the Wilson loop was discussed in a more general framework by $\mathrm{O}$. Alvarez [3]. In the current renewal of interest in quantum string theory it has become clear that the Polyakov path integral provides an efficient, manifestly covariant description of string theory at the critical dimension [4-6]. The basic objects of the modern $S$-matrix formulation are the on-shell multiloop amplitudes defined by means of the Polyakov functional integral over surfaces with prescribed topology and with vertex functionals corresponding to the ingoing and outgoing on-shell particle states [7]. The advantage of this approach is that the Polyakov functional measure is reduced via the Faddeev-Popov procedure to the uniquely determined measure on moduli space [3-6]. As a result, on-shell amplitudes can be written as finite dimensional integrals over moduli space of expressions made up of the known functions [8]. In the past few years, important progress has been made in the investigation of these amplitudes, especially in the case of the closed string [4-9]. Within the $S$-matrix formulation, the sum over bordered surfaces appears in the functional integral representation of the on-shell open string amplitudes [10]. 
Although the $S$-matrix approach provides a full description of physical processes, there are some questions of interest (i.e. the string vacuum, general coordinate invariance, and low energy limits) which seem hard to answer within this prescription. An alternative approach based on Alvarez' paper [3] has been proposed by Cohen et al. [11]. The general idea of this approach is to consider the functional integral over surfaces (with a given topology and endowed with an intrinsic metric), connecting prescribed contours $k_{1}, \ldots, k_{N}$ in the target space:

$$
A^{\text {off }}\left[k_{1}, \ldots, k_{N}\right]=\int_{\mathscr{M}_{M}} \mathscr{D} g \int_{\mathscr{E}_{M}^{k}} \mathscr{D} x\left(\operatorname{Vol} \mathscr{D}_{M} \times \operatorname{Vol} \mathscr{W}_{M}\right)^{-1} e^{-s[g, x]} .
$$

In the case of a closed string the model manifold $M$ is a two-dimensional oriented manifold with $N$-holes and $h$-handles and the functional integral (1.1) is interpreted as an $h$-loop $N$-states off-shell closed string amplitude.

In contrast to the functional integral representation of on-shell amplitudes, the evaluation (= definition) of (1.1) by the F-P procedure causes some problems. In general the F-P method works only when the number of nondynamical variables in a functional integral is equal to the number of parameters of a gauge group. To be more precise, it can happen that nondynamical degrees of freedom become dynamical due to an anomaly [1], but it is not of concern in our case $(d=26)$. A simple counting shows that there are too many nondynamical variables in (1.1). One can resolve this problem by imposing some $\mathscr{D}_{M} \odot \mathscr{W}_{M}$-invariant boundary condition on the space of fields $\mathscr{M}_{M} \times \mathscr{E}_{M}^{k}$. Such a boundary condition has been proposed by Alvarez [3],

$$
n_{g}^{a} t^{b} \partial_{a} x^{\mu} \partial_{b} x^{\mu}=0
$$

The problem with this condition is that we cannot integrate over $x$-variables. If we consider (1.2) for a fixed metric $g$ as a condition for $x$, the submanifold of $\mathscr{E}_{M}^{k}$ determined by (1.2) is not an affine space nor a sum of various affine spaces and the functional integral over $x$ is not Gaussian and therefore untractable. In the literature [3,11-17] the calculation (= definition) of the functional(1.1) is based on a modification of the result obtained in the case of closed surfaces:

$$
A^{\text {off }}\left[k_{1}, \ldots, k_{N}\right]=\prod_{i=1} \int_{\mathscr{D} M} \mathscr{D} \gamma_{i} \int_{\left[T_{M}^{*}\right]}[d t]\left(\operatorname{det}^{\prime} P^{+} P\right)^{1 / 2}(\operatorname{det} \mathscr{L})^{-13} e^{-S\left[g, x_{c l}\right]}
$$

The determinant of $P^{+} P$, as well as the finite dimensional determinants included in the measure $[d t]$ on a fundamental domain $\left[T_{M}^{\#}\right]$ in the reduced Teichmüller space [25], are evaluated with the mixed boundary conditions [3]. The determinant of the Laplace-Beltrami operator $\mathscr{L}_{g}$ is evaluated with the Dirichlet boundary conditions, and $x_{c l}$ denotes the solution of the equation $\mathscr{L}_{g} x_{c l}=0$ with the boundary conditions $\left.x_{c l}^{\mu}\right|_{\partial M}=k_{i}^{\mu} \circ \gamma_{i}$.

The expression (1.3) is unsatisfactory for several reasons. First of all the mixed boundary conditions do not follow from the boundary conditions (1.2) and therefore cannot cure the problem of nondynamical variables in (1.1) mentioned above. This is just the origin of the averaging over boundary reparametrizations in (1.3). In contrast to other functional measures in Polyakov's theory the functional measure $\mathscr{D} \gamma^{i}$ remains undetermined and is an untractable formal symbol. This problem disappears for pointlike strings, i.e. for the semi- off-shell amplitudes obtained by shrinking the contours $k_{i}$ to points $[11,17]$. In this case the expression 
(1.3) leads to the expected results $[12-14,17]$. Let us also note that even if it is possible to provide a precise meaning to the averaging over boundary reparametrizations the resulting expression should be interpreted as an off-shell amplitude sandwiched between projectors on the space of diff-invariant states [12]. It is clear that such objects are insufficient to build up the Feynman rules for the interacting string field theory.

There are some other evidences [18-22] that the off-shell amplitudes in the string theory should be gauge dependent and therefore the averaging over reparametrizations in (1.3) seems to be spurious. The arguments in [21, 22] are mainly of a string field theory nature, while the derivations in $[18,19]$ are based on the BRST extension of the string hamiltonian and the proper time representation of the propagator. In [20] another derivation of the off-shell string propagator within the Batalin-Fradkin-Vilkoviski phase space path integral approach has been proposed. In all these cases the original geometrical interpretation of the Polyakov path integral is lost.

The aim of this paper is to construct a consistent covariant functional integral representation of off-shell open string amplitudes. The general idea of the approach presented here is to consider the off-shell amplitudes as functionals either on the space $\mathscr{C}_{L}^{26}$ of contours in $\mathbb{R}^{26}$ endowed with the intrinsic onedimensional metric or on the space $\overline{\mathscr{C}}_{L}^{26}=\mathscr{C}_{L}^{26} / \mathbb{R}_{+}$. Such choices of variables in the off-shell formulation of string theory are justified by the structure of the space of string wave functionals in the first quantized theory in the covariant gauge.

In Sect. 2 the consistency of the F-P procedure is discussed in the case of the functional integral representation of on-shell open string amplitudes. This section has an introductory character and the majority of the presented materials is not new. The only novelty is an "integrated" version of the mixed Alvarez boundary conditions which play an essential role in further considerations.

In Sect. 3 the covariant functional first quantization of the open bosonic string in the position representation is carried out, according to the Feynman functional quantization scheme [23] adapted to the case of the gauge theories with the reparametrization invariance. The general ideas of this quantization scheme are presented in Subsect. 3.1. In Subsect. 3.2 two different spaces $\overline{\mathfrak{H}}^{\text {off }}, \mathfrak{H}^{\text {off }}$ of open string wave functionals are introduced. They lead to different formulations of the first quantized string. The functional integral representations of the open string propagators in both formulations are proposed and evaluated in Sect. 3.3. It was shown that the consistency of these representations requires an additional stage of the gauge fixing consisting in a choice of an appropriate subbundle of allowed metrics in the space of string trajectories. This gauge freedom is related to the freedom of choice of the open string hamiltonian. The considerations in Sect. 3 do not provide a full description of the first quantized string. The complete formulation requires the BRST extension of the propagator and of the space of wave functionals. This problem is beyond the scope of this paper and will be presented elsewhere. Nevertheless, the results of Sect. 3 allow us to generalize the functional integral representation of the propagator to the case of arbitrary offshell open string amplitudes. Such a generalization is presented in Sect. 4. Conclusions and a discussion of directions of further work are included in Sect. 5. 


\section{The On-Shell Open String Amplitudes}

In order to give a meaning (via the F-P procedure) to the Polyakov functional integral over surfaces with a boundary it is necessary to specify appropriate boundary conditions [3]. In the case of on-shell open string amplitudes the boundary conditions for the variable $x: M \rightarrow \mathbb{R}^{d}$ are a natural generalization (for an arbitrary bordered Riemann surface $M$ ) of the string edge conditions. They follow from the variation of the classical BDHP [20] action:

$$
\left.n_{g}^{a} \partial_{a} x^{\mu}\right|_{\partial M}=0 \text {. }
$$

$n_{g}$ denotes the normal inward direction to the boundary $\partial M$ with respect to the metric $g$ on $M$. This condition is invariant under the action of the semidirect product $\mathscr{D}_{M}^{0} \odot \mathscr{W}_{M}$ of the group $\mathscr{D}_{M}^{0}$ of all diffeomorphisms of $M$ connected with the identity and the group $\mathscr{W}_{M}$ of conformal deformations of metrics on $M$. Throughout the whole paper we will deal only with $\mathscr{D}_{M}^{0}$. (The residual modular invariance can be taken into account by restricting the final integral over the reduced Teichmüller space [21] to a fundamental domain of the discrete mapping class group $\left.\mathscr{D}_{M} / \mathscr{D}_{M}^{0}\right)$. In order to simplify the notation we shall write in the sequel $\mathscr{D}_{M}$ instead of $\mathscr{D}_{M}^{0}$.

We would like to define a partition function for the open string by analogy with the closed string case [4-6]:

$$
Z_{M}^{0}=\int_{\mathscr{M}_{M}} \mathscr{D} g \int_{\delta_{M^{R}}}\left(\int_{\mathscr{D}_{M}} \mathscr{D} f \times \int_{\mathscr{W}_{M}} \mathscr{D} \phi\right)^{-1} e^{-S[g, x]},
$$

where $\mathscr{M}_{M}$ is the space of all metrics on $M, \mathscr{E}_{M}^{n_{g}}$ - the space of mappings $x: M \rightarrow \mathbb{R}^{d}$ fulfilling the boundary condition (2.1); $S[g, x]$ denotes the BDHP action for the bosonic string. According to Polyakov's ideas $[1,28$.$] , the functional measures in$ (2.2) are treated as infinite dimensional volume forms related to the ultralocal Riemannian structures $H(\mid), E^{g}(\mid), H^{g}(\mid), W^{g}(\mid)$ defined on $\mathscr{M}_{M}, \mathscr{E}_{M}^{n_{\mathcal{Z}}}, \mathscr{D}_{M}, \mathscr{W}_{M}$, respectively:

$$
\begin{aligned}
& M_{g}\left(\delta g \mid \delta g^{\prime}\right) \equiv \int_{M} \sqrt{g} d^{2} z g^{a c} g^{b d} \delta g_{a b} \delta g_{c d}^{\prime}, \quad \delta g, \delta g^{\prime} \in \mathscr{T}_{g} \mathscr{M}_{M}, \\
& E_{x}^{g}\left(\delta x \mid \delta x^{\prime}\right) \equiv \int_{M} \sqrt{g} d^{2} z \delta x^{\mu} \delta x^{\prime \mu}, \quad \delta x, \delta x^{\prime} \in \mathscr{T}_{x} \mathscr{E}_{M}^{n_{g}} \approx \mathscr{E}_{M}^{n_{g}}, \\
& H_{i d}^{g}\left(\delta f \mid \delta f^{\prime}\right) \equiv \int_{M} \sqrt{g} d^{2} z g_{a b} \delta f^{a} \delta f^{\prime b}, \quad \delta f, \delta f^{\prime} \in \mathscr{T}_{i d} \mathscr{D}_{M}^{0}, \\
& W_{\phi}^{g}\left(\delta \phi \mid \delta \phi^{\prime}\right) \int_{M} \sqrt{g} d^{2} z \delta \phi \delta \phi^{\prime}, \quad \delta \phi, \delta \phi^{\prime} \in \mathscr{T}_{\phi} \mathscr{W}_{M} \approx \mathscr{W}_{M} .
\end{aligned}
$$

Since the quotient space $\mathscr{M}_{M} /\left(\mathscr{D}_{M} \times \mathscr{W}_{M}\right)$ is homeomorphic to the reduced Teichmüller space $T_{M}^{\#}$ of the surface $M$ [25] one can expect that the functional integral (2.2) reduces (via the F-P procedure) to a finite dimensional one over $T_{M}^{\#}$.

The integration over $\mathscr{E}_{M}^{n_{\mathscr{Z}}}$ in (2.2) is Gaussian and yields the $\mathscr{D}_{M}$-invariant functional on $\mathscr{M}_{M}$ :

$$
\int_{\mathscr{E}_{M}^{M} g} \mathscr{D} x e^{-S[g, x]}=\left(\frac{\operatorname{det}_{N}^{\prime} \mathscr{L}_{g}}{\int_{M} \sqrt{g} d^{2} z}\right)^{-\mathrm{d} / 2},
$$


where $\operatorname{det}_{N}^{\prime} \mathscr{L}_{g}$ denotes the determinant of the Laplace-Beltrami operator acting on the space of scalar functions with the Neumann boundary conditions.

In the conformal gauge the F-P procedure with respect to the group $\mathscr{D}_{M}$ is based on the splitting of the space $\mathscr{T}_{g} \mathscr{M}_{M}$ tangent to $\mathscr{M}_{M}$ at every point $g \in \mathscr{M}_{M}$,

$$
\mathscr{T}_{g} \mathscr{M}_{M}=\mathscr{H}_{g} \oplus \mathscr{K}_{g},
$$

into the space $\mathscr{H}_{g}$ of traceless symmetric tensors $\delta h_{a b}$ and the space $\mathscr{K}_{g}$ of tensors proportional to the metric $\delta \phi g_{a b}\left(\delta \phi \in \mathscr{W}_{M}\right)$.

The F-P operator $\Delta_{g}^{(+)}=P_{g}^{+} P_{g}$ is defined by means of the conformal Lie derivative operator $P_{g}[3,22]$ :

$$
P_{g}: \mathscr{T}_{i d} \mathscr{D}_{M} \rightarrow \mathscr{H}_{g}, \quad\left(P_{g} \delta f\right)_{a b}=\nabla_{a} \delta f_{b}+\nabla_{b} \delta f_{a}-g_{a b} \nabla_{c} \delta f^{c},
$$

and its formal adjoint $P_{g}^{+}$,

$$
P_{g}^{+}: \mathscr{H}_{g} \rightarrow \mathscr{T}_{i d} \mathscr{D}_{M}^{0}, \quad\left(P_{g}^{+} \delta h\right)_{a}=-2 \nabla^{b} \delta h_{a b},
$$

where $\mathscr{T}_{i d} \mathscr{D}_{M}$ denotes the space tangent to the group $\mathscr{D}_{M}$ at the identity.

As it was pointed out by Alvarez [3] the determinant of $P_{g}^{+} P_{g}$ can be evaluated if the operators $\Delta_{g}^{(+)}$and $\Delta_{g}^{(-)}=P_{g} P_{g}^{+}$are self-adjoint positively semidefined elliptic operators. This requirement, together with the additional one concerning the relation between the spectra of $\Delta_{g}^{(+)}$and $\Delta_{g}^{(-)}$, leads to the following general structure of the admissible boundary conditions on $\partial M[3]$ :

$$
\mathscr{B}_{1} \delta f=0, \quad \mathscr{B}_{2} \delta h=0, \quad \mathscr{B}_{2}\left(P_{g} \delta f\right)=0, \quad \mathscr{B}_{1}\left(P_{g}^{+} \delta h\right)=0,
$$

where $\mathscr{B}_{1}, \mathscr{B}_{2}$ are local linear relations not involving derivatives for which the integral

$$
\int_{\partial M} d s n^{a} \delta h_{a b} \delta f^{b}
$$

vanishes.

The space $\mathscr{T}_{i d} \mathscr{D}_{M}$ consists of vector fields $\delta f^{a}$ an $M$ with the boundary condition $\left.n_{a} \delta f^{a}\right|_{\partial M}=0$. This condition has the unique extension to the system (2.10):

$$
\begin{gathered}
n_{a} \delta f^{a}=0, \\
t^{a} n^{b} \delta h_{a b}=0, \\
t^{a} n^{b}\left(P_{a} \delta f\right)_{a b}=0, \\
n^{\alpha}\left(P_{g}^{+} \delta h\right)_{a}=0 .
\end{gathered}
$$

From now on the system above will be called on $A$-type system.

The additional boundary conditions mean that in the functional integral (2.2) we cannot integrate over the whole space $\mathscr{M}_{M}$ but rather over a submanifold of $\mathscr{M}_{M}$ the tangent space of which is determined by (2.12), (2.14). Similarly, the condition (2.13) leads to a restriction of the diffeomorphisms group.

In order to construct an appropriate submanifold of $\mathscr{M}_{M}$ let us first recall some basic facts about the doubling construction [13]. For a given oriented 2-dimensional surface $M$ with boundary we consider its copy $\bar{M}$ with the opposite orientation (the mirror image). The double $M_{D}$ of $M$ is the orientable closed 
manifold obtained from $M$ and $\bar{M}$ by gluing about the corresponding points on the appropriate boundaries. With this construction $M$ and $\bar{M}$ become bordered submanifolds of $M_{D}$ and $\partial M=M \cap \bar{M}$ is a one-dimensional submanifold of $M_{D}$. We say that an orientation reversing diffeomorphism $i$ of $M_{D}$ is an involution of $M_{D}$ if its set of fixed points is $\partial M$ and $i^{2}=\mathrm{id}_{M_{D}}$. Note that the existence of at least one such involution follows immediately from the definition of $M_{D}$. Equivalently one can define the doubling as a closed manifold with involution. For every involution $i$ on $M_{D}$ there is the uniquely determined invariant direction $n_{i}$ on $\partial M$ :

$$
i_{*} n_{i}=-n_{i} \text {. }
$$

A metric $g$ on $M_{D}$ will be called $i$-symmetric if the involution $i$ is an isometry of $g:$

$$
i^{*} g=g \text {. }
$$

It is clear that for an $i$-symmetric metric $g$ the normal direction $n_{g}$ determined by $g$ on $\partial M$ coincides with the invariant direction of $i$,

$$
n_{i}^{a} t^{b} g_{a b}^{s} \overline{\partial M} 0
$$

Furthermore, for any $i$-symmetric vector field $V^{(+)}$on $M_{D}$,

$$
V^{(+)}=i_{*} V^{(+)},
$$

and for any $i$-antysymmetric vector field $V^{(-)}$on $M_{D}$,

$$
V^{(-)}=-i_{*} V^{(-)}
$$

we have the identities:

$$
\begin{aligned}
& n_{i}^{a} V_{a}^{(+)} \underset{\partial \bar{M}}{=} 0, \quad t^{a} n_{i}^{b} \nabla_{b} V_{a}^{(+)} \underset{\partial \overline{\partial M}}{=} 0, \\
& t^{a} V_{a}^{(-)} \underset{\partial M}{\overline{\partial M}} 0, \quad n_{i}^{a} n_{i}^{b} \nabla_{b} V_{a}^{(-)} \underset{\partial \bar{M}}{\overline{\bar{y}}} 0,
\end{aligned}
$$

where $t^{a}$ denotes the unit vector tangent to $\partial M$. Note that $\partial M$ is geodesic with respect to the metric $g$.

Let us now consider a metric $g$ on $M$ and define the metric $g^{s}$ on $M_{D}$ by setting

$$
\left.g^{s}\right|_{\bar{M}}=i^{*} g,\left.\quad g^{s}\right|_{M}=g,
$$

where $i$ is some involution of $M_{D}$. The resulting metric $g^{s}$, in general, will not be continous and differentiable across $\partial M$. The coincidence of the normal direction $n_{g s}$ with $n_{i}$ is a necessary condition for the metric (2.19) to be continuous. Equations (2.17), (2.18) provide conditions for $\left(C^{1}\right)$ differentiability. The conditions for continuity of higher order derivatives of $g$ can be formulated by relations on $\partial M$ involving higher order covariant derivatives of (anti-) symmetric vector fields.

For a given involution $i$ on $M_{D}$ we define the space $\mathscr{M}_{M}^{i, 1}$ as a space of metrics admitting a $C^{1}$ extension to $i$-symmetric metrics on $M_{D}$. We are interested in the space $\mathscr{T}_{g} \mathscr{M}_{M}^{i}$ tangent to $\mathscr{M}_{M}^{i}$ at $g \in \mathscr{M}_{M}^{i}$. This space consists of symmetric covariant 
tensors $\delta g_{a b}$ fulfilling the boundary conditions:

$$
\begin{array}{r}
n_{i}^{a} t^{a} \delta g_{a b} \underset{\overline{\partial M}}{ } 0, \\
n^{a} \nabla^{b} \delta g_{a b}=0 \\
n^{a} \nabla_{a}\left(g^{b c} \delta g_{b c}\right)=0 \\
\overline{\partial M}
\end{array}
$$

The conditions above can be easily derived by the variation of the conditions (2.16-2.18). The important feature of the boundary conditions (2.20) is that they are compatible with the orthogonal decomposition (2.7):

$$
\begin{gathered}
\mathscr{T}_{g} \mathscr{M}_{M}^{i}=\mathscr{H}_{g}^{i} \oplus \mathscr{K}_{g}^{i}, \\
\mathscr{H}_{g}^{i}=\left\{\delta h_{a b} \in \mathscr{H}_{g}: n_{i}^{a} t^{b} \delta h_{a b}=0, n_{i}^{a} \nabla^{b} \delta h_{a b}=\overline{\overline{\partial M}} 0\right\}, \\
\mathscr{K}_{g}^{i}=\left\{\delta \tau g_{a b} \in \mathscr{K}_{g}: n_{i}^{a} \nabla_{a} \delta \tau \overline{\partial M} 0\right\} .
\end{gathered}
$$

The space $\mathscr{H}_{g}^{i}$ is just the space of symmetric tensors determined by the boundary conditions (2.12), (2.14), therefore we should replace $\mathscr{M}_{M}$ by $\mathscr{M}_{M}^{i}$ in the functional integral (2.2). But $\mathscr{M}_{M}^{i}$ is not invariant under the action of $\mathscr{D}_{M} \odot \mathscr{W}_{M}$, so we must restrict the gauge group too. Fortunately, this can be done consistently with the boundary conditions (2.13).

A diffeomorphism $f$ of $M_{D}$ will be called $i$-symmetric if it commutes with the involution $i$ :

$$
i \circ f \circ i=f .
$$

It is clear that if $g$ and $f$ are $i$-symmetric on $M_{D}$ then $f^{*} g$ is $i$-symmetric too. For $f \in \mathscr{D}_{M}$ let us introduce the diffeomorphism $f_{s}$ of $M_{D}$ defined by

$$
\left.f_{s}\right|_{M}=f,\left.\quad f_{s}\right|_{\bar{M}}=i \circ f \circ i .
$$

The diffeomorphism $f_{s}$ constructed in this way is $i$-symmetric and continuous for all $f \in \mathscr{D}_{M}$. It follows from (2.22) and the uniqueness of the invariant direction $n_{i}$ that the equation:

$$
f_{s *} n_{i} \overline{\overline{\partial M}} n_{i}
$$

is a necessery condition for $f_{s}$ to have continuous first derivative.

We define the subgroup $\mathscr{D}_{M}^{i, 1} \in \mathscr{D}_{M}$ as the group of all diffeomorphisms of $M$ which admits the $C^{1}$ extension to $i$-symmetric diffeomorphisms of $M_{D}$. The space $\mathscr{T}_{i d} \mathscr{D}_{M}^{i, 1}$ consists of all vector fields on $M$ satisfying the boundary conditions (2.11) and (2.13).

In a similar way one can obtain the restricted group of conformal deformations:

$$
\mathscr{W}_{M}^{i, 1}=\left\{\phi \in \mathscr{W}_{M}: n_{i}^{a} \partial_{a} \phi \underset{\overline{\partial M}}{0}\right\}
$$


Resuming, the imposition of the $A$-type boundary conditions necessary to ensure the consistency of the F-P procedure is equivalent to the restriction of $\mathscr{M}_{M}$ and $\mathscr{D}_{M} \odot \mathscr{W}_{M}$ in the definition of the partition function to $\mathscr{M}_{M}^{i, 1}$ and $\mathscr{D}_{M}^{i, 1} \odot \mathscr{W}_{M}^{i, 1}$ respectively. There is, however, some technical problem related to the geometry of the action of the group of diffeomorphisms on the space of metrics. In fact, $\mathscr{M}_{M}^{i, 1}$ is invariant under the action of the group $\mathscr{D}_{M}^{i, 2}$ (diffeomorphisms which admit continuous, up to the second derivative, $i$-symmetric extension on $M_{D}$ ) but not under the action of $\mathscr{D}_{M}^{i, 1}$. To avoid this problem we can consider the spaces $\mathscr{M}_{M}^{i, \infty}$, $\mathscr{D}_{M}^{i, \infty}, \mathscr{W}_{M}^{i, \infty}$ defined by means of smooth $i$-symmetric extensions on the doubling. This is possible because for a smooth $i$-symmetric metric $g$ on $M_{D}$ the operators $P_{g}^{+} P_{g}$ and $P_{g} P_{g}^{+}$are elliptic and positively defined and therefore they have smooth eigenfunctions which can be symmetrically divided into $i$-symmetric and $i$-antysymmetric parts. It is clear that the $i$-symmetric part provides the solution of the $A$-type boundary value problem for the operators $P_{g}^{+} P_{g}$ on $\mathscr{T}_{i d} \mathscr{D}_{M}^{i, \infty}$ and $P_{g} P_{g}^{+}$ on $\mathscr{H}_{M}^{i, \infty}$. Therefore, for the evaluation of the F-P determinant it is inessential in which class of spaces we work (provided that the boundary condition up to the first derivative is taken into account). Note that the quotient space $\mathscr{M}_{M}^{i, \infty} /\left(\mathscr{D}_{M}^{i, \infty} \odot \mathscr{W}_{M}^{i, \infty}\right)$ is the relative Teichmüller space $T_{M}^{R}$ of $M$ [26] which is isomorphic to the reduced Teichmüller space $T_{M}^{\#}$ [27]. The relation between the $A$-type boundary value problem and the doubling construction was first pointed out by Blau and Clements in the case of constant curvature metrics and used in the evaluation of the off-shell closed string amplitudes [13].

We arrive at the following definition of the open string partition function:

$$
Z_{M}^{0, i}=\int_{\mathcal{M}^{i, \infty}} \mathscr{D} g \int_{\mathscr{E}_{M}^{i}} \mathscr{D} x\left(\operatorname{Vol} \mathscr{D}_{M}^{i, \infty} \times \operatorname{Vol} \mathscr{W}_{M}^{i, \infty}\right)^{-1} e^{-S[g, x]},
$$

where $\mathscr{E}_{M}^{i}=\mathscr{E}_{M}^{n_{g}}$.

The new definition above depends on some arbitrary chosen involution $i$ on $M$ (in particular it depends on the invariant direction $n_{i}$ ). Using the F-P method one can check by the explicit calculation of (2.23) that $Z_{M}^{0, i}$ is in fact independent of $i$. It can also be seen in a more heuristic way. For another involution $i^{\prime}$ on $M_{D}$ we have the smooth diffeomorphism $f=\left.i^{\prime} \circ i\right|_{M}$. Since the measure and the action $S[g, x]$ in (2.23) are explicitly $\mathscr{D}_{M}$-invariant, the change of variables $(g, x) \rightarrow\left(f^{*} g, f^{*} x\right)$ results only in the change of integration domain $\mathscr{M}_{M}^{i, \infty} \times \mathscr{E}_{M}^{i} \rightarrow \mathscr{M}_{M}^{i^{\prime}, \infty} \times \mathscr{E}_{M}^{i^{\prime}}$ and we obtain $Z_{M}^{0 i}=Z_{M}^{0 i^{\prime}}$.

For the functional integral (2.23) one can apply the F-P procedure exactly as in the case of closed surfaces [28]. Let us stress that the boundary conditions for the F-P operator follow from our choice of domain of integration. Moreover, at every stage of evaluation of (2.23) (i.e. for every $g \in \mathscr{M}_{M}^{i, \infty}$ ) one can use the doubling construction [13]. It allows us to treat the problem of the local and global conformal anomaly as in the case of the closed string [6]. The evaluation of (2.23) proceeds along the standard lines and the final form of the partition function in the conformal gauge $g=e^{\phi} \hat{g}_{t}$ is as follows:

$$
Z_{M}^{0}=\int_{\left[T_{M}^{*}\right]} d^{6 h-6+3 b} t \operatorname{det} M_{\hat{g}^{t}}\left(\delta \psi_{i} \mid \bar{\chi}_{k}\right)\left(\frac{\sum \operatorname{det}_{A} P_{\hat{g}^{t}} P_{\hat{g}^{t}}}{\operatorname{det} H^{+}\left(P_{\hat{g}^{t}}^{+}\right)}\right)\left(\frac{\operatorname{det}_{N^{2}} \mathscr{L}_{\hat{g}^{t}}}{\int_{M} \sqrt{\hat{g}^{t}} d^{2} z}\right)^{-13},
$$


where $M$ is a surface with $h$-handles and $b$-boundaries, $\left\{\delta \psi_{i}\right\}_{i=1}^{6 h-6-3 b}$ denotes an arbitrary basis in $\operatorname{Ker} P_{\hat{g}^{t}}^{+}$and

$$
\begin{gathered}
\left(\delta \bar{\chi}_{j}\right)_{a b}=\left(\frac{\partial}{\partial t^{j}} \hat{g}_{a b}^{t}-\frac{1}{2} \hat{g}_{a b}^{t} \hat{g}^{t c d} \frac{\partial}{\partial t^{j}} \hat{g}_{c d}^{t}\right), \\
H\left(P_{\hat{g}^{t}}^{+}\right)_{i j}=M_{\hat{g}^{t}}\left(\delta \psi_{i}, \delta \psi_{j}\right) .
\end{gathered}
$$

The determinants of $P_{g}^{+} P_{g}$ and $\mathscr{L}_{g}$ are evaluated with the $A$-type and Neumann boundary conditions, respectively. The formula $(2.24)$ is the starting point to discuss the on-shell open string amplitudes [10].

\section{The Covariant Functional Quantization of the Open Bosonic String}

\subsection{General Ideas}

Within the Feynman prescription of the covariant functional quantization of nondegenerate Lagrangians [23] the space $H$ of wave functions consists of complex valued functions on the space $Q$ of boundary conditions $\left\{q_{i}\right\}_{i=1}^{n}$ of the trajectories of a classical system with the inner product

$$
\int_{Q} d^{n} q \bar{\psi}(q) \phi(q)
$$

The central object of this approach is the integral kernel (with respect to (3.1)) of the evolution operator expressed in terms of a functional integral over trajectories.

The degenerate Lagrangian case requires changes in both stages of the scheme above. For the gauge theories the space of boundary conditions divides into classes of physically equivalent conditions. Therefore, the wave functions representing the physical states should be constant in the equivalence classes. These classes can be described as orbits of some group $\mathscr{G}$ (acting on the space of initial conditions) induced by the group of gauge transformations (acting on the space of trajectories). Accordingly, the "physical" wave functions can be described as $\mathscr{G}$-invariant functions. Roughly speaking, the number of parameters of the group $\mathscr{G}$ is equal to the number of first class constraints linear in momenta present in the Dirac analysis [29] of a system considered. The restriction to the space of wave functions with some symmetry in the covariant prescription is then equivalent in the canonical quantization to the restriction to the subspace of states annihilated by the corresponding constraint operators.

For gauge theories without reparametrization invariance all first class constraints are linear in momenta and can be taken into account by the requirement of an appropriate invariance of wave functions. The quantization scheme is completed in this case by evaluating the integral kernel of the evolution operator determined as a functional integral over classes of gauge equivalent trajectories. Let us note that the problem of constructing an appropriate inner product in the space of symmetric wave functions is not trivial.

The important feature of gauge theories with reparametrization invariance is the appearance of first class constraints quadratic in momenta. Moreover, since in this case the gauge transformations connect trajectories starting and ending at different times the wave functions corresponding to the physical states should be 
time independent. In fact, due to the vanishing of the canonical Hamiltonian for such theories the Schrödinger equation is trivial and the whole dynamics is described by the constraint equations [30]. The integral kernel of the evolution operator is simply the delta function but there exists a corresponding object defined by the functional integral over trajectories - the propagator which has the quantum mechanical interpretation as the integral kernel of the operator inverse to the operator of a quadratic in momenta constraint. More precisely, such interpretation is valid only when one can consistently solve all linear in momenta constraints. With a part of these constraints unsolved the propagator acquires an additional contribution from the Stückelberg and the ghost fields.

In the next section we will show how these general ideas of covariant functional quantization can be applied in the case of the open bosonic string described by the BDHP action [24].

\subsection{The Space of the Open String Wave Functionals}

In general the open string trajectory is described by the triplet $(M, g, x)$, where $M$ is a rectangle-like 2-dimensional manifold, $g$ is a metric on $M$ and $x$ is a mapping from $M$ into the target space $\mathbb{R}^{d}$. The general gauge transformations include the Weyl rescalings of the metric and the diffeomorphisms $f: M \rightarrow M^{\prime}$ (which can change the manifold $M$ ). The first stage of the gauge fixing in the presented formulation consists in the choice of some fixed 2-dimensional manifold $M$ (diffeomorphic to the rectangle). With this gauge the space of string trajectories and the space of gauge transformations reduce to the space $\mathscr{M}_{M} \times \mathscr{E}_{M}^{d}$ and to the group $\mathscr{D}_{M} \odot \mathscr{W}_{M}$, respectively.

Let us note that other gauges are also possible. In the local formulation (in terms of infinitesimal metric variations) they were discussed by Redlih [21]. The general idea of this approach is to consider the metric variations generated by diffeomorphisms moving the boundaries of $M$. In the global formulation it requires a notion of path integral over the space of 2-dimensional rectangle-like manifolds and in particular a $\mathscr{D}$-invariant Riemannian structure on this space. Realization of this program leads to complicated constructions and will not be discussed here.

We will call two pairs of the opposite sides of $M$ the "timelike" $(T)$ and "spacelike" $(S)$ boundaries of $M$ (Fig. 1a). In order to specify the boundary conditions along the $T$-boundaries of $M$, we will use the doubling $M_{D}$ of $M$ (Fig. 1b) with some involution $i$ on $M_{D}$. As it follows from considerations of the previous section the space of string trajectories is defined as the cartesian product $\mathscr{T}_{M}^{i}=\mathscr{M}_{M}^{i} \times \mathscr{E}_{M}^{i}$ of the space $\mathscr{M}_{M}^{i}$ of the metrics on $M$ with smooth $i$-symmetric extensions on $M_{D}$ and the space $\mathscr{E}_{M}^{i}$ of the mappings with the Neumann boundary conditions along the $T$-boundaries of $M$. In addition, we require that the boundaries of $M$ be perpendicular one to another at all corners of $M$ with respect to the metrics $g \in \mathscr{M}_{M}^{i}$ [17]. The group of the gauge transformations $\mathscr{D}_{M}^{i} \odot \mathscr{W}_{M}^{i}$ consists of the diffeomorphisms $f: M \rightarrow M$ admitting smooth $i$-symmetric extensions on $M_{D}$ and the functions $\phi: M \rightarrow \mathbb{R}$ with the Neumann boundary conditions along the $T$-boundaries.

Let us consider the space $\mathscr{A}$ of the boundary conditions of string trajectories. Within our ( $M$-fixed) gauge the initial (final) boundary conditions for an open 

where

$$
I_{i}: \sum_{i} \rightarrow M, \quad(i \rightarrow f)
$$

are the inclusions of the initial $\Sigma_{i}$ and the final $\Sigma_{f}$ boundaries of $M$ (henceforth $(i \rightarrow f)$ denotes the same formula for $f$ indices). All possible initial conditions form the cartesian product $\mathscr{M}_{\Sigma_{i}} \times \mathscr{E}_{\Sigma_{i}}^{d}$ of the space $\mathscr{M}_{\Sigma_{i}}$ of all einbeins on $\Sigma_{i}$ and the space $\mathscr{E}_{\Sigma_{i}}^{d}$ of all mappings $x_{i}: \Sigma_{i} \rightarrow \mathbb{R}^{d}$ satisfying the Neumann boundary conditions at the ends of $L$. Accordingly, the final boundary conditions form the space $\mathscr{M}_{\Sigma_{f}} \times \mathscr{E}_{\Sigma_{f}}^{d}$. Both spaces are independent of the special parametrization of $M, \Sigma_{i}$, and $\Sigma_{f}$. However, it should be possible to compare the initial and the final conditions. This can be done by introducing the "reference" space $\mathscr{M}_{L} \times \mathscr{E}_{L}^{d}$ together with the isomorphisms

$$
\sigma_{i}^{*}: \mathscr{M}_{\Sigma_{i}} \times \mathscr{E}_{\Sigma_{i}}^{d} \rightarrow \mathscr{M}_{L} \times \mathscr{E}_{L}^{d}, \quad(i \rightarrow f)
$$

induced by the diffeomorphisms: $\sigma_{i}: L \rightarrow \Sigma_{i},(i \rightarrow f)$. The choice of some "reference" interval $L(L=[0,1])$ can be regarded as the first stage of the 1-dimensional part of gauge fixing in our formulation.

Restricting oneself to the space of string wave functionals on $\mathscr{M}_{L} \times \mathscr{E}_{L}^{\text {d }}$ gives part of the linear in momenta constraints already solved. The remaining unsolved part is related to the residual induced gauge transformations on $\mathscr{M}_{L} \times \mathscr{E}_{L}^{d}$. They form the semidirect product $\mathscr{D}_{L} \odot \mathscr{W}_{L}$ of the group $\mathscr{D}_{L}$ of all orientation preserving diffeomorphisms $\gamma: L \rightarrow L$ and the group $\mathscr{W}_{L}$ of all real valued functions $\tilde{\phi}$ on $L$ with the Neumann boundary conditions $(\dot{\tilde{\phi}}(0)=\tilde{\phi}(1)=0)$. The action of $\mathscr{D}_{L} \odot \mathscr{W}_{L}$ on $\mathscr{M}_{L}$ $\times \mathscr{E}_{L}^{d}$ has the following form:

$$
\mathscr{M}_{L} \times \mathscr{E}_{L}^{d} \ni(e, \tilde{x}) \stackrel{(\gamma, \tilde{\phi}) \in \mathscr{D}_{L} \odot \mathscr{W}_{L}}{\longrightarrow}\left(\exp (\tilde{\phi}) \gamma^{*} e, \gamma^{*} \tilde{x}\right) \in \mathscr{M}_{L} \times \mathscr{E}_{L}^{d}
$$

The identification of the initial and final boundary conditions of string trajectories with points in $\mathscr{M}_{L} \times \mathscr{E}_{L}^{d}$ manifestly depends on an arbitrary choice of the parametrizations $\sigma_{i}, \sigma_{f}$ (3.3). One can overcome this problem choosing the quotient $\mathscr{C}_{L}^{d}=\left(\mathscr{M}_{L} \times \mathscr{E}_{L}^{d}\right) / \mathscr{D}_{L}$ as the space of boundary conditions for string trajectories. The space $\mathscr{C}_{L}^{d}$ has a simple geometrical interpretation - it is the space of all metrized open contours in $\mathbb{R}^{d}$. For every string trajectory we define the initial $c_{i}$ and the final $c_{f}$ boundary conditions by

$$
c_{i}=\Pi_{\mathscr{C}}\left(\sigma_{i}^{*} e_{i}, \sigma_{i}^{*} x_{i}\right), \quad(i \rightarrow f),
$$

where $\Pi_{\mathscr{C}}$ denotes the canonical projection in the principal bundle

$$
\begin{aligned}
\mathscr{D}_{L} \rightarrow \mathscr{M}_{L} \times \mathscr{E}_{L}^{d} & \\
& \downarrow \\
& \left(\mathscr{M}_{L} \times \mathscr{E}_{L}^{d}\right) / \mathscr{D}_{L}=\mathscr{C}_{L}^{d} \approx \mathbb{R}_{+} \times \mathscr{E}_{L}^{d} .
\end{aligned}
$$

We define the space $\mathfrak{H}^{\text {off }}$ of off-shell string wave functionals as the space of all functionals on $\mathscr{C}_{L}^{d}$. One way of description (parametrization) of the space $\mathfrak{H}^{\text {off }}$ is offered by the Faddeev-Popov method. One can consider $\mathfrak{H}^{\text {off }}$ as the space of $\mathscr{D}_{L}$-invariant functionals on $\mathscr{M}_{L} \times \mathscr{E}_{L}^{d}$, which are completely determined by their values on some gauge slice $\mathscr{S}_{L}$ related to a global section of the bundle (3.4). Note 
that in the case of the open string the bundle (3.4) is trivial (there is no Gribov ambiguity).

In the sequel we restrict ourselves to a special subclass of gauges which can be called 1-dimensional conformal gauges and are determined by the gauge slices:

$$
\mathscr{S}_{L}^{\hat{e}}=\left\{(e, \tilde{x}) \in \mathscr{M}_{L} \times \mathscr{E}_{L}^{d}: e=\lambda \hat{e}, \lambda \in \mathbb{R}_{+}\right\} .
$$

This restriction is the 1-dimensional part of the second stage of gauge fixing in our formulation. In this gauge the string wave functional $\Psi[c]$ can be regarded as the functional $\Psi_{\hat{e}}[\alpha, \tilde{x}]=\Psi[\alpha \hat{e}, \tilde{x}]$ on the space $\mathbb{R}_{+} \times \mathscr{E}_{L}^{d}$.

Let us now turn to the description of the residual gauge invariance in the space $\mathfrak{H}^{\text {off }}$ related to the "unsolved" $\mathscr{W}_{L}$-invariance in $\mathscr{M}_{L} \times \mathscr{E}_{L}^{d}$. Due to the semidirect product structure of the group $\mathscr{D}_{L} \odot \mathscr{W}_{L}$ the action of $\mathscr{W}_{L}$ on $\mathscr{M}_{L} \times \mathscr{E}_{L}^{d}$ does not reduce to a well defined action on $\mathscr{C}_{L}^{d}$ (with the exception of the 1-dimensional subgroup $\mathbb{R}_{+}\left(\mathscr{W}_{L}\right.$ of constant rescalings of einbeins which commute with $\left.\mathscr{D}_{L}\right)$.

With respect to this problem the gauges (3.5) are very special. For a fixed $\hat{e}$ one can construct the subgroup $\widetilde{D}_{L}^{e} \subset \mathscr{D}_{L} \odot \mathscr{W}_{L}$ defined by

$$
\widetilde{D}_{L}^{\hat{e}}=\left\{(\tilde{\phi}, \tilde{\gamma}) \in \mathscr{D}_{L} \odot \mathscr{W}_{L}: e^{\tilde{\phi}_{\gamma}} \hat{e}=\hat{e}\right\} .
$$

The direct product $\mathbb{R}_{+} \times \tilde{\mathscr{D}}_{L}^{\hat{e}}$ preserves the gauge slice $\mathscr{S}_{L}^{\hat{e}}$ and describes the residual gauge invariance in the gauge (3.5). In fact the group $\mathscr{D}_{L} \odot \mathscr{W}_{L}$ can be decomposed as the semidirect product

$$
\mathscr{D}_{L} \odot \mathscr{W}_{L}=\mathscr{D}_{L} \odot\left(\mathbb{R}_{+} \times \widetilde{\mathscr{D}}_{L}^{\hat{e}}\right),
$$

and for every $\hat{e}$ we have

$$
\mathscr{S}_{\mathbf{L}}^{\hat{e}} /\left(\mathbb{R}_{+} \times \widetilde{\mathscr{D}}_{\mathbf{L}}^{\hat{e}}\right) \approx \mathscr{K}^{d}
$$

The residual gauge invariance in $\mathscr{C}^{d}$ can be also described in a gauge independent way. Let us consider the space $\mathscr{M}_{L} \times \widetilde{\mathscr{D}}_{L}$, where $\widetilde{\mathscr{D}}_{L}$ denotes the group of the orientation preserving diffeomorphisms of $L$. The action of the group $\mathbb{R}_{+}$ $\times \mathscr{D}_{L}$ on $\mathscr{M}_{L} \times \widetilde{D}_{L}$ defined by

$$
\mathscr{M}_{L} \times \widetilde{\mathscr{D}}_{L} \ni(e, \tilde{\gamma}) \stackrel{(\lambda, \gamma) \in \mathbb{R}_{+} \times \mathscr{D}_{L}}{\longrightarrow}\left(\lambda \gamma^{*} e, \gamma^{-1} \tilde{\gamma} \gamma\right) \in \mathscr{M}_{L} \times \widetilde{\mathscr{D}}_{L}
$$

induces the principal fibre bundle structure:

$$
\begin{aligned}
& \mathbb{R}_{+} \times \mathscr{D}_{L} \rightarrow \mathscr{M}_{L} \times \widetilde{\mathscr{D}}_{L} \\
& \downarrow \Pi_{\mathscr{g}} \\
& \mathscr{G}_{L} \equiv\left(\mathscr{M}_{L} \times \widetilde{\mathscr{D}}_{L}\right) /\left(\mathbb{R}_{+} \times \mathscr{D}_{L}\right) .
\end{aligned}
$$

Let us consider the family of global sections of this bundle determined by the gauge slices:

$$
\left\{(e, \tilde{\gamma}) \in \mathscr{M}_{L} \times \tilde{D}_{L}: e=\hat{e}\right\} .
$$

We define the group structure in $\mathscr{G}_{L}$ :

$$
\mathscr{G}_{L} \times \mathscr{G}_{L} \ni\left(\Gamma, \Gamma^{\prime}\right) \rightarrow \Gamma \Gamma^{\prime} \equiv \Pi_{\mathscr{G}}\left(\hat{e}, \tilde{\gamma} \circ \tilde{\gamma}^{\prime}\right) \in \mathscr{G}_{L},
$$


where $\Gamma=\Pi_{\mathscr{G}}(\hat{e}, \tilde{\gamma}), \Gamma^{\prime}=\Pi_{\mathscr{G}}\left(\hat{e}, \tilde{\gamma}^{\prime}\right)$. The right action of $\mathscr{G}$ on $\mathscr{C}_{L}^{d}$ is defined by:

$$
\mathscr{C}_{L}^{d} \ni c \stackrel{\Gamma \in \mathscr{G}}{\longrightarrow} \Gamma c \equiv \Pi_{\mathscr{C}}(\alpha \hat{e}, \tilde{x} \circ \tilde{\gamma}) \in \mathscr{C}^{d},
$$

where $c=\Pi_{\mathscr{C}}(\alpha \hat{e}, \tilde{x})$ and $\Gamma=\Pi_{\mathscr{G}}(\hat{e}, \tilde{\gamma})$.

Both definitions above are $\hat{e}$-independent. From (3.7) it easily follows that $\mathscr{G}_{L}$ is isomorphic to $\mathscr{D}_{L}$. We also have

$$
\mathscr{C}_{L}^{d} / \mathbb{R}_{+} \times \mathscr{G}_{L}=\mathscr{K}^{d} .
$$

The subspace $\mathfrak{H}_{p h}^{\text {off }} \subset \mathfrak{H}^{\text {off }}$ of the "off-shell physical" wave functionals corresponding to the string states annihilated by all constraints linear in momenta can then be defined as the subspace of $\mathscr{G}_{L}$-invariant functionals on $\mathscr{C}_{L}^{d}$.

Let us introduce another space of string boundary conditions:

$$
\overline{\mathscr{C}}_{L}^{d}=\mathscr{C}_{L}^{d} / \mathbb{R}_{+}=\left(\mathscr{M}_{L} \times \mathscr{E}_{L}^{d}\right) /\left(\mathscr{D}_{L} \times \mathbb{R}_{+}\right) .
$$

As in the case of $\mathscr{C}_{L}^{d}$, for every string trajectory $(g, x) \in \mathscr{M}_{M}^{i} \times \mathscr{E}_{M}^{d}$ one can define two points in $\overline{\mathscr{C}}_{L}^{d}$ as the initial $\bar{c}_{i}$ and the final $\bar{c}_{f}$ boundary conditions independent of any parametrization of $M, \Sigma_{i}$, and $\Sigma_{f}$. We will denote the space of string wave functionals defined on $\overline{\mathscr{C}}_{L}^{d}$ or, equivalently, the subspace of $\mathbb{R}_{+}$-invariant functionals on $\mathscr{C}_{L}^{d}$ by $\overline{\mathfrak{H}}^{\text {off }}$. The parametrization of the space based on the FaddeevPopov method requires global sections in the bundle:

$$
\begin{aligned}
\mathbb{R}_{+} \times \mathscr{D}_{L} \rightarrow \mathscr{M}_{L} \times \mathscr{E}_{L}^{d} \\
\downarrow_{\overline{\mathscr{\ell}}} \\
\overline{\mathscr{C}}_{L}^{d} \approx \mathscr{E}_{L}^{d} .
\end{aligned}
$$

We will use the 1-dimensional conformal gauges:

$$
\overline{\mathscr{T}}_{L}^{\hat{e}}=\left\{(e, \tilde{x}) \in \mathscr{M}_{L} \times \mathscr{E}_{L}^{d}, e=\hat{e}\right\} .
$$

The residual gauge transformations are described in $\overline{\mathscr{C}}_{L}^{d}$ by the action of the group $\mathscr{G}_{L}$.

There also exist gauge independent parametrizations of the spaces $\mathscr{C}_{L}^{d}, \overline{\mathscr{C}}_{L}^{d}$. For a given einbein $e \in \mathscr{M}_{L}$, let us consider the 1-dimensional Laplace-Beltrami operator

$$
\mathscr{L}_{e}=-\frac{1}{e} \frac{d}{d \sigma} \frac{1}{e} \frac{d}{d \sigma}
$$

acting on the space of scalar functions on $L$ satisfying the Neumann boundary conditions. Let us denote by $\left\{\psi_{n}^{e}\right\}_{n>0}$ the complete basis of all normalized eigenfunctions of $\mathscr{L}_{\hat{e}}$ :

$$
\begin{gathered}
\mathscr{L}_{e} \psi_{n}^{e}=\lambda_{n} \psi_{n}^{e},\left.\quad \frac{d}{d \sigma} \psi_{n}^{e}\right|_{\sigma=0,1}=0 \\
\int_{L} e \psi_{n}^{e} \psi_{m}^{e} d \sigma=\delta_{n, m}, \quad n, m=0,1 .
\end{gathered}
$$


We define the mapping $\mathscr{P}$ from the space $\mathscr{M}_{L} \times \mathscr{E}_{L}^{d}$ onto the space $\Lambda^{d}$ of infinite sequences of modes by

$$
\begin{gathered}
\mathscr{P}: \mathscr{M}_{L} \times \mathscr{E}_{L}^{d} \ni(e, \tilde{x}) \rightarrow\left(\ell, c_{0}^{\mu}, c_{1}^{\mu}, \ldots\right) \in \Lambda^{d}, \\
\ell=\int_{L} e d \sigma, \\
c_{n}^{\mu}=\int_{L} e \psi_{x}^{e} x^{\mu} d \sigma, \quad n=0,1 .
\end{gathered}
$$

It follows from the completeness of $\left\{\psi_{n}^{e}\right\}_{n>0}$ and the invariance of $\mathscr{L}_{\hat{e}}\left(\psi_{n}^{\gamma^{*} e}=\gamma^{*} \psi_{n}^{e}\right)$ that $\mathscr{S}$ is constant along $\mathscr{D}_{L}$-orbits in $\mathscr{M}_{L} \times \mathscr{E}_{L}^{d}$ and reduces to the 1-1 mapping $\mathscr{P}: \mathscr{C}_{L}^{d} \rightarrow \Lambda^{d}$. In this parametrization the $\mathbb{R}_{+}$action on $\mathscr{C}^{d}$ has the following form:

$$
\left(\ell, c_{0}^{\mu}, c_{1}^{\mu}, \ldots\right) \stackrel{\lambda \in \mathbb{R}_{+}}{\longrightarrow}\left(\lambda \ell, \lambda^{1 / 2} c_{0}^{\mu}, \lambda^{1 / 2} c_{1}^{\mu}, \ldots\right) .
$$

The corresponding gauge independent parametrization of $\overline{\mathscr{C}}^{d}$ is defined by

$$
\overline{\mathscr{P}}: \mathscr{M}_{L} \times \mathscr{E}_{L}^{d} \ni(e, \tilde{x}) \rightarrow\left(\bar{c}_{0}^{\mu}, \bar{c}_{1}^{\mu}, \ldots\right) \in \bar{\Lambda}^{d}, \quad \bar{c}_{n}^{\mu}=\ell^{-1 / 2} c_{n}^{\mu}, \quad n=0,1, \ldots .
$$

The next task is to construct an inner product in the space $\mathfrak{G}_{p h}^{\text {off }}$. It requires some notion of integration of $\mathscr{D}_{L} \odot \mathscr{W}_{L}$-invariant functionals over $\mathscr{M}_{L} \times \mathscr{E}_{L}^{d}$. Applying the geometrical framework of functional integration of invariant objects one should start with some ultralocal, invariant Riemannian structure on $\mathscr{M}_{L} \times \mathscr{E}_{L}^{d}$. Just like in the case of the space of string trajectories there are no $\mathscr{D}_{L} \odot \mathscr{W}_{L}$-invariant, ultralocal structures on $\mathscr{M}_{L} \times \mathscr{E}_{L}^{d}$ (one can achieve either $\mathscr{D}_{L}$ - or $\mathscr{W}_{L}$-invariance). The $\mathscr{D}_{L}$-invariant metric structure $G(\mid)$ on $\mathscr{M}_{L} \times \mathscr{E}_{L}^{d}$ is well known from the functional quantization of the spinless particle $[11,28]$, where $\mathscr{M}_{L} \times \mathscr{E}_{L}^{d}$ plays the role of the space of trajectories. We have [28]

$$
G_{(e, \tilde{x})}\left(\delta e, \delta \tilde{x} \mid \delta e^{\prime}, \delta \tilde{x}^{\prime}\right)=\int_{L} \frac{\delta e \delta e^{\prime}}{e} d s+\int_{L} e \delta x^{\mu} \delta x^{\prime \mu} d s .
$$

We define the inner product (.) in $\mathfrak{H}_{\text {oh }}^{\text {off }}$ by

$$
\left(\Psi, \Psi^{\prime}\right)=\int_{\mathcal{M}_{L} \times \mathscr{E}^{d} L}(e, \tilde{x})\left(\operatorname{Vol}_{e} \overline{\mathscr{D}}_{L}\right)^{-1}\left(\operatorname{Vol}_{e} \mathscr{W}_{L}\right)^{-1} \bar{\Psi}[e, \tilde{x}] \Psi^{\prime}[e, \tilde{x}],
$$

where the functional measure $\mathscr{D}(e, \tilde{x})$ is related to the metric structure $G(\mid)$. In the gauge (3.5) the standard application of the Faddeev-Popov method with respect to the group $\mathscr{D}_{L}$ yields

$$
\left(\Psi \mid \Psi^{\prime}\right)=\hat{\ell} \int_{0}^{\infty} d \alpha \int_{\tilde{E}_{L}} \mathscr{D}^{\alpha \hat{e}} \tilde{x}\left(\operatorname{Vol}_{\alpha \hat{e}} \mathscr{W}_{L}\right)^{-1} \bar{\Psi}_{\hat{e}}[\alpha, \tilde{x}] \Psi_{\hat{e}}^{\prime}[\alpha, \tilde{x}],
$$

where $\hat{\ell}=\int_{L} \hat{e} d \sigma$ and the functional measure $\mathscr{D}^{\alpha \hat{x}} \tilde{x}$ is related to the metric structure $E^{\alpha \hat{e}}(\mid)$ on $\mathscr{E}_{L}^{26}$ defined as follows:

$$
E_{\tilde{x}}^{\alpha \hat{e}}\left(\delta \tilde{x} \mid \delta \tilde{x}^{\prime}\right)=\int_{L} \alpha \hat{e} \delta \tilde{x} \delta \tilde{x}^{\prime} d s .
$$

The functional measure in the inner product (3.13) is not $\mathbb{R}_{+} \times \mathscr{G}_{L}$-invariant and cannot be reduced by the Faddeev-Popov method to an ultralocal measure on the quotient spaces $\overline{\mathscr{C}}_{L}^{d}=\mathscr{C}_{L}^{d} / \mathbb{R}_{+}, \mathscr{K}^{d}=\overline{\mathscr{C}}_{L}^{d} / \mathscr{G}_{L}$. 


\subsection{The Open String Propagator}

According to the general scheme of the functional quantization presented in Subsect. 3.1 the string propagator $P\left[\bar{c}_{i}, \bar{c}_{f}\right]$ in the space $\overline{\mathfrak{H}}^{\text {off }}$ is defined as a path integral over the space $\overline{\mathscr{T}}_{M}^{i}(i, f)$ of the string trajectories starting at the "initial" class of metrized contours $\bar{c}_{i} \in \overline{\mathscr{C}}_{L}^{d}$ and ending at the "final" one $\bar{c}_{f} \in \overline{\mathscr{C}}_{L}^{d}$. In the parametrization of $\overline{\mathscr{C}}_{L}^{d}$ determined by the gauge fixing $\overline{\mathscr{S}}_{L}^{\hat{e}}(3.8)$ one can formulate these conditions in the following way:

$$
\Pi_{\overline{\mathscr{C}}}\left(\sigma_{i}^{*} e_{i}, \sigma_{i}^{*} x_{i}\right)=\Pi_{\overline{\mathscr{C}}}\left(\hat{e}, \tilde{x}_{i}\right)=\bar{c}_{i}, \quad(i \rightarrow f) .
$$

Note that the conditions (3.14) are independent of the choice of parametrization $\sigma_{i}: L \rightarrow \Sigma_{i}, \sigma_{f}: L \rightarrow \Sigma_{f}$ and of the choice of gauge fixing in $\overline{\mathscr{C}}_{L}^{d}$.

The boundary conditions (3.14) are $\mathscr{D}_{M}^{i}$-invariant, and one can expect that in the "evaluation" of the propagator $P\left[\bar{c}_{i}, \bar{c}_{f}\right]$ the Faddeev-Popov method should be applied for the group $\mathscr{D}_{M}^{i}$. As was discussed in Sect. 2 the requirement of consistency of this step leads to some restrictions on the space of metrics and gauge transformations. In our case the boundary conditions along the $T$-boundaries of $M$ fulfill this requirement. It is convenient to derive the corresponding conditions along the $S$-boundaries $\Sigma_{i}, \Sigma_{f}$ of $M$, before we give an appropriate definition of the string propagator. Repeating the considerations of Sect. 2 we obtain the space of open string trajectories $\mathscr{T}_{M}^{i j}=\mathscr{M}_{M}^{i j} \times \mathscr{E}_{M}^{d}$, where $\mathscr{M}_{M}^{i j}$ is the subspace of $\mathscr{M}_{M}^{i}$ consisting of all metrics admitting $j$-symmetric extensions on $M^{D} . M^{D}$ denotes the doubling of $M$ with respect to the "spacelike" boundaries and $j$ is an arbitrarily chosen involution on $M^{D}$ (Fig. 1c). The corresponding restriction of the gauge group $\mathscr{D}_{M}^{i} \odot \mathscr{W}_{M}^{i}$ we will denote by $\mathscr{D}_{M}^{i j} \odot \mathscr{W}_{M}^{i j}$.

Since the conditions (3.14) are not invariant under the action of the whole group $\mathscr{W}_{M}^{i j}$, the gauge invariance which should be taken into account in a functional integral representation of the propagator is related to the subgroup $\mathscr{D}_{M}^{i j} \odot \bar{W}_{M}^{i j} \subset \mathscr{D}_{M}^{i j} \odot \mathscr{W}_{M}^{i j}$, where $\overline{\mathscr{W}}_{M}^{i j}$ denotes the subgroup of $\mathscr{W}_{M}^{i j}$ consisting of functions which are constant on the $S$-boundaries $\Sigma_{i}, \Sigma_{f}$. It leads to the following formal expression for the open string propagator:

$$
P\left[\bar{c}_{i}, \bar{c}_{f}\right]=\int_{\overline{\mathscr{T}}_{M}^{i j}(i, f)} \mathscr{D}(g, x)\left(\int_{\mathscr{D}_{M}^{i j}} \mathscr{D} f\right)^{-1}\left(\int_{\overline{\mathscr{W}}_{M}^{j}} \mathscr{D} \phi\right)^{-1} e^{-S[g, x]},
$$

where the functional measures $\mathscr{D}(g, x), \mathscr{D} f, \mathscr{D} \phi$ are related to the Riemannian structures defined by $(2.3,4),(2.5)$, and $(2.6)$ respectively. Note that there are too many nondynamical variables in the path integral (3.15), so one can expect that this expression should be modified. We will return to this problem after the discussion of the F-P procedure.

Using the formal generalization of the Fubini theorem [28] one can replace the integration over $\overline{\mathscr{T}}_{M}^{i j}(i, f)$ by the integration over the subspaces of a fixed $g$ and then over the manifold $\mathscr{M}_{M}^{i j}$ :

$$
P\left[\bar{c}_{i}, \bar{c}_{f}\right]=\int_{\mathscr{M}_{M}^{i j}} \mathscr{D} g\left(\operatorname{Vol} \mathscr{D}_{M}^{i j}\right)^{-1}\left(\operatorname{Vol} \overline{\mathscr{W}}_{M}^{i j}\right)^{-1} \int_{\mathscr{E}_{M}^{g}(i, f)} \mathscr{D} x e^{-S[g, x]} .
$$

$\mathscr{E}_{M}^{g}(i, f)$ denotes the affine space of mappings $x: M \rightarrow \mathbb{R}^{d}$ fulfilling the boundary conditions:

$$
x_{i}=\tilde{x}_{i} \circ \gamma_{i}[g, \hat{e}], \quad(i \rightarrow f)
$$


where for every $g$ the diffeomorphisms

$$
\gamma_{i}[g, \hat{e}]: \Sigma_{i} \rightarrow L, \quad(i \rightarrow f),
$$

are uniquely determined by the equations

$$
\text { const }_{i} \times e_{i}=\gamma_{i}^{*}[g, \hat{e}] \hat{e}, \quad(i \rightarrow f) .
$$

Since for $\gamma \in \mathscr{D}_{L}$

$$
\gamma_{i}\left[g, \gamma^{*} \hat{e}\right]=\gamma^{-1} \circ \gamma_{i}[g, \hat{e}], \quad(i \rightarrow f)
$$

and

$$
\gamma_{i}[g, \text { const } \times \hat{e}]=\gamma_{i}[g, \hat{e}], \quad(i \rightarrow f),
$$

the boundary conditions (3.17) are independent of the choice of gauge $\overline{\mathscr{S}}_{L}^{\hat{e}}$ in $\overline{\mathscr{C}}^{d}$.

The functional integral over $\mathscr{E}_{M}^{g}(i, f)$ is Gaussian and can be evaluated:

$$
\int_{\mathscr{G}(i, f)} \mathscr{D} x e^{-S[g, x]}=\left(\operatorname{det}_{D N} \mathscr{L}_{g}\right)^{-d / 2} e^{-S\left[g, x_{i}^{f}[g]\right]},
$$

where $\mathscr{L}_{g}$ denotes the 2-dimensional Laplace-Beltrami operator acting on the space of scalar functions on $M$ with the Dirichlet boundary conditions along the $S$-boundaries and the Neumann ones along the $T$-boundaries. $x_{i}^{f}[g]$ is a solution of the boundary value problem

$$
\begin{gathered}
\mathscr{L}_{g} x_{i}^{f}[g]=0, \\
x_{i}^{f}[g] \circ I_{i}=\tilde{x}_{i} \circ \gamma_{i}[g, \hat{e}], \quad(i \rightarrow f), \\
\left(n^{i}\right)^{a} \partial_{a} x_{i}^{f}[g]=0 \quad \text { on } \quad \partial M \backslash\left(\Sigma_{i} \cap \Sigma_{f}\right) .
\end{gathered}
$$

The right-hand side of formula (3.20) is a $\mathscr{D}_{M}^{i j}$-invariant functional of $g$ so one can apply the standard Faddeev-Popov method to the path integral:

$$
P\left[\bar{c}_{i}, \bar{c}_{f}\right]=\int_{\mathscr{M}_{M}^{i j}} \mathscr{D} g\left(\operatorname{Vol} \mathscr{D}_{M}^{i j}\right)^{-1}\left(\operatorname{Vol} \overline{\mathscr{W}}_{M}^{i j}\right)^{-1}\left(\operatorname{det}_{D N} \mathscr{L}_{g}\right)^{-d / 2} e^{-S\left[g, x_{i}^{f}[g]\right]}
$$

with respect to the group $\mathscr{D}_{M}^{i j}$.

In order to construct a conformal gauge slice let us consider the principal fibre bundle [25-27]:

$$
\begin{aligned}
\mathscr{D}_{M}^{i j} \odot \mathscr{W}_{M}^{i j} \rightarrow & \mathscr{M}_{M}^{i j} \\
& { }^{\Pi_{T}} \\
& \mathscr{M}_{M}^{i j} /\left(\mathscr{D}_{M}^{i j} \odot \mathscr{W}_{M}^{i j}\right)=T_{M}^{R}=T_{M}^{\#} .
\end{aligned}
$$

In the case of a rectangle the relative (reduced) Teichmüller space $T_{M}^{R}\left(T_{M}^{\#}\right)$ is isomorphic with $\mathbb{R}_{+}$and there exists a global smooth section of the bundle (3.23):

$$
\Xi: T_{M}^{R} \ni t \rightarrow g^{t} \in \mathscr{M}_{M}^{i j} \text {. }
$$

Using the section $\Xi$ one can construct the global conformal section $\Xi^{c}$ of the bundle:

$$
\begin{gathered}
\mathscr{D}_{M}^{i j} \rightarrow \mathscr{M}_{M}^{i j} \\
\downarrow \\
\mathscr{M}_{M}^{i j} / \mathscr{D}_{M}^{i j}
\end{gathered}
$$


determined by the conformal gauge slice $\mathscr{S}_{\mathbb{\Xi}}^{c}=\Xi^{c}\left(\mathscr{M}_{M}^{i j} / \mathscr{D}_{M}^{i j}\right)$ :

$$
\mathscr{S}_{\Xi}^{c}=\left\{\hat{g} \in \mathscr{M}_{M}^{i j}: \hat{g}=e^{\phi} g^{t}, \phi \in \mathscr{W}_{M}^{i j}, t \in T_{M}^{R}\right\} .
$$

The application of the F-P method for the functional integral (3.22) in the gauge fixing (3.25) together with the change of variables $\mathscr{S}_{\mathcal{E}}^{c} \ni \hat{\mathrm{g}} \rightarrow(t, \phi) \in \mathbb{R}^{+} \times \mathscr{W}_{M}^{i j}$ yields

$$
\begin{aligned}
P\left[\bar{c}_{i}, \bar{c}_{f}\right]= & \int_{0}^{\infty} d t M_{g^{t}}(\delta \psi \mid \delta \bar{\chi}) \int_{W_{i j}} \widetilde{\mathscr{D}} \phi\left(\int_{\hat{W}_{i j}} \mathscr{D} \phi\right)^{-1}\left(\frac{\operatorname{det}_{A A} P_{\hat{g}}^{+} P_{\hat{g}}}{H\left(P_{\hat{g}}^{+}\right)}\right)^{1 / 2} \\
& \times\left(\operatorname{det}_{D N} \mathscr{L}_{\hat{g}}\right)^{-d / 2} e^{-S\left[\hat{g}, x_{i}^{f}[\hat{g}]\right]},
\end{aligned}
$$

where all symbols are defined as in Sect. 2. The index $A A$ in the symbol of determinant of $P_{\hat{g}}^{+} P_{\hat{g}}$ means that the $A$-type boundary conditions have been chosen on the $S$ - and $T$-boundaries of $M$. Note that there are no conformal Killing vector fields on $M$ with the $A$-type boundary conditions [17]. The functional measure $\widetilde{\mathscr{D}} \phi$ in (3.26) is related to the nonconstant Riemannian structure $\tilde{W}_{\phi}^{g^{t}}(\mid)$ on $\mathscr{W}_{M}^{i j}$,

$$
\tilde{W}_{\phi}\left(\delta \phi \mid \delta \phi^{\prime}\right)=\int_{M} e^{\phi} \sqrt{g} t d^{2} z \delta \phi \delta \phi^{\prime} .
$$

As was shown in [17], in the case of bordered surfaces with corners the conformal anomaly does not vanish even in the critical dimension. It is due to the non-vanishing corner contribution which in $d=26$ has the form $\exp \left[\frac{5}{8} \sum_{i} \phi\left(\bar{z}_{i}\right)\right]$ (the sum is taken over all corners $z_{i}$ of $M$ ). Therefore in $d=26$ one can rewrite (3.26) in the following form:

$$
\begin{aligned}
P\left[\bar{c}_{i}, \bar{c}_{f}\right]= & \int_{0}^{\infty} d t M_{g^{t}}(\delta \psi \mid \delta \bar{\chi}) \int_{W_{i j}} \tilde{\mathscr{D}} \phi\left(\int_{\tilde{W}_{i j}^{i j}} \mathscr{D} \phi\right)^{-1}\left(\frac{\operatorname{det}_{A A} P_{\hat{g}}^{+} P_{\hat{g}}}{H\left(P_{\hat{g}}^{+}\right)}\right)^{1 / 2}\left(\operatorname{det}_{D N} \mathscr{L}_{\hat{g}}\right)^{-13} \\
& \times \exp \left(\frac{5}{8} \sum_{i} \phi\left(z_{i}\right)-S\left[e^{\phi} g^{t}, x_{i}^{i}\left[e^{\phi} g^{t}\right]\right]\right) .
\end{aligned}
$$

In the formula above a drawback of the definition (3.15) of the propagator becomes more visible. Even at the critical dimension the integration over "conformal factor" does not decouple. There are two independent sources of this difficulty. First of all, as a result of our choice of boundary conditions for trajectories (3.14) the maximal symmetry group of the "classical action" $S\left[g, x_{i}^{f}[g]\right]$ is $\mathscr{D}_{M}^{i j} \odot \overline{\mathscr{W}}_{M}^{i j}$ (and not $\mathscr{D}_{M}^{i j} \odot \mathscr{W}_{M}^{i j}$ ). Secondly, due to the corner anomaly the symmetry group of the integrand in (3.27) reduces further to the group $\mathscr{D}_{M}^{i j} \odot \overline{\mathscr{W}}_{M}^{i j}$, where $\overline{\mathscr{W}}_{M}^{i j}$ consists of all functions $\phi \in \overline{\mathscr{W}}_{M}^{i j}$ satisfying the condition: $\sum \phi\left(z_{i}\right)=0$.

Note that the symmetry properties of $S\left[g, x_{i}^{f}[g]\right]$ are similar for open and closed strings while the corner anomaly is a specific feature of the open string. For this reason it is convenient to consider the difficulty with (3.27) in two steps. Firstly, we assume that there is no corner anomaly. With this assumption all problems are due to the integration over the whole group $\mathscr{W}_{M}^{i j}$ in (3.27). This is the reflection of the problem mentioned above with nondynamical variables in the ("too large") space of trajectories $\overline{\mathscr{T}}_{M}^{i j}(i, f)$. It follows from formulae (3.22) and (3.27) that an 
appropriate space of trajectories (i.e. a space for which it is possible to define the path integral (3.15) by Gaussian integrals and by the F-P method) should lead to the space of metrics $\mathscr{B}_{M} \subset \mathscr{M}_{M}^{i j}$ which has the structure of a subbundle of (3.23) with the structure group $\mathscr{D}_{M}^{i j} \odot \overline{\mathscr{W}}_{M}^{i j}$.

Let us denote by $\mathfrak{B}_{M}$ the space of all subbundles $\mathscr{B}_{M}$,

$$
\begin{aligned}
\mathscr{D}_{M}^{i j} \odot \overline{\mathscr{W}}_{M}^{i j} \rightarrow \mathscr{B}_{M} & \\
& \downarrow_{\mathscr{B}} \\
\mathscr{B}_{M} /\left(\mathscr{D}_{M}^{i j} \odot \overline{\mathscr{W}}_{M}^{i j}\right)=T_{M}^{R} &
\end{aligned}
$$

of the bundle (3.23). Note that fixing some "reference" section of (3.23) one can parametrize the space $\mathfrak{B}_{M}$ by the space of functions:

$$
T_{M}^{R} \ni t \rightarrow\left(\phi_{i}^{t}, \phi_{f}^{t}\right) \in \mathscr{W}_{M} \times \mathscr{W}_{M}
$$

For every $\mathscr{B}_{M} \in \mathfrak{B}_{M}$ we define the functional

$$
P\left[\bar{c}_{i}, \bar{c}_{f} \mid \mathscr{B}_{M}\right]=\int_{\overline{\mathscr{T}}_{M}^{i j}\left(i, f \mid \mathscr{B}_{M}\right)} \mathscr{D}(g, x)\left(\int_{\mathscr{D}_{M}^{i j}} \mathscr{D} f\right)^{-1}\left(\int_{\bar{W}_{M}^{i j}} \mathscr{D} \phi\right)^{-1} e^{-S[g, x]},
$$

where

$$
\overline{\mathscr{T}}_{M}^{i j}\left(i, f \mid \mathscr{B}_{M}\right)=\left\{(g, x) \in \overline{\mathscr{T}}_{M}^{i j}(i, f): g \in \mathscr{B}_{M}\right\} .
$$

The expression (3.29) fulfills the consistency requirement of the F-P method, and the functional $P\left[\bar{c}_{i}, \bar{c}_{f} \mid \mathscr{B}_{M}\right]$ can be evaluated at the critical dimension:

$$
P\left[\bar{c}_{i}, \bar{c}_{f} \mid \mathscr{B}_{M}\right]=\int_{0}^{\infty} d t M_{g^{t}}(\delta \psi \mid \delta \bar{\chi})\left(\frac{\operatorname{det}_{A A} P_{g^{t}}^{+} P_{g^{t}}}{H\left(P_{g^{t}}^{+}\right)}\right)^{1 / 2}\left(\operatorname{det}_{D N} \mathscr{L}_{g^{t}}\right)^{-13} e^{-S\left[g^{t}, x_{i}^{f}\left[g^{t}\right]\right]},
$$

where $\Xi: T_{M}^{R} \ni t \rightarrow g^{t} \in \mathscr{B}_{M}$ is a global section of the subbundle $\mathscr{B}_{M}$.

Let us consider the functional (3.30) in the special case of the subbundle $\mathscr{B}_{M}^{0}$ constructed from the subspace $\mathscr{M}_{M 0}^{i j}$ of all metrics $g \in \mathscr{M}_{M}^{i j}$ with zero scalar curvature by the action of the group $\overline{\mathscr{W}}_{M}^{i j}$. Note that this subbundle is in a way "natural" since its definition is independent of any parametrization of the Teichmüller space and does not require any additional structure on $M$. In the case of $\mathscr{B}_{M}^{0}$ an explicit construction of the global section $\Xi_{0}: T_{M}^{R} \ni t \rightarrow g_{0}^{t} \in \mathscr{B}_{M}^{0} \subset \mathscr{M}_{M}^{i j}$ is especially easy. In the parametrization $\left(z^{0}, z^{1}\right) \in[0,1] \times[0,1] \approx M$ of the rectangle $M$ one can define $\Xi_{0}$ by $[11,17]$ :

$$
T_{M}^{R} \approx \mathbb{R}_{+} \ni t \rightarrow g_{0}^{t}=\left(\begin{array}{ll}
t^{2} & 0 \\
0 & 1
\end{array}\right) .
$$

Taking into account (3.21), (3.30) and proceeding as in [11] we have

$$
P\left[\bar{c}_{i}, \bar{c}_{f} \mid \mathscr{B}_{M}^{0}\right]=\mathrm{const} \int_{0}^{t} d t e^{\pi t}\left[\Pi\left(1-e^{i 2 \pi n t}\right)\right]^{-12} t^{-13} e^{-S\left[g_{0}^{t}, x_{i}^{f}\left[g_{0}^{t}\right]\right]},
$$

where

$$
\begin{aligned}
S\left[g_{0}^{t}, x_{i}^{f}\left[g_{0}^{t}\right]\right] & =W\left[\bar{c}_{i}, \bar{c}_{f} \mid t\right] \\
& =\frac{1}{4 \pi \alpha}\left[\frac{\left(\bar{c}_{i}^{0}-\bar{c}_{f}^{0}\right)^{2}}{t}+\sum_{n=1}^{\infty} \frac{2 \pi n}{\operatorname{sh}(\pi n t)}\left[\left(\bar{c}_{i}^{n^{2}}+c_{f}^{n^{2}}\right) \operatorname{ch}(\pi n t)-2 \bar{c}_{i}^{n} \bar{c}_{f}^{n}\right]\right]
\end{aligned}
$$


and

$$
\bar{c}_{i}^{k}=\ell_{i}^{-1 / 2} c_{i}^{k}, \quad k=0,1,2, \ldots, \quad(i \rightarrow f)
$$

denote the gauge invariant parametrization (3.12) of $\bar{c}_{i}, \bar{c}_{i} \in \overline{\mathscr{C}}_{L}^{d}$.

Since every subbundle $\mathscr{B}_{M} \in \mathfrak{B}_{M}$ can be obtained from another one by a vertical automorphism of the bundle (3.23) we are able to express the functional $P\left[\bar{c}_{i}, \bar{c}_{f} \mid \mathscr{B}_{M}\right]$ in terms of $\mathscr{B}_{M}^{0}$. For every global section $\Xi: T_{M}^{R} \ni t \rightarrow g^{t} \in \mathscr{B}_{M}$ there exists a function $T_{M}^{R} \ni t \rightarrow \phi^{t} \in \mathscr{W}_{M}^{i j}$ and a section $\bar{\Xi}_{0}: T_{M}^{R} \ni t \rightarrow \bar{g}_{0}^{t} \in \mathscr{B}_{M}^{0}$ for which

$$
g^{t}=\exp \left(\phi^{t}\right) \bar{g}^{t} .
$$

Let $\bar{c}_{i}=\Pi_{\tilde{\mathscr{b}}}\left(\hat{e}, \tilde{x}_{i}\right),(i \rightarrow f)$. From the $\mathscr{W}_{M}^{i j}$-invariance of the BDHP action we have

$$
S\left[g^{t}, x_{i}^{f}\left[g^{t}\right]\right]=S\left[\bar{g}^{t}, x_{i}^{f}\left[g^{t}\right]\right],
$$

where $x_{i}^{f}\left[g^{t}\right]$ is the solution of the boundary value problem (3.21) with $g=g^{t}$ and with the boundary conditions:

$$
x_{i}^{f}\left[g^{t}\right] \circ I_{i}=\tilde{x}_{i} \circ \gamma_{i}\left[g^{t}, \hat{e}\right], \quad(i \rightarrow f) .
$$

Since

$$
\mathscr{L}_{\bar{g}^{t}} x_{i}^{f}\left[g^{t}\right]=\mathscr{L}_{g^{t}} x_{i}^{f}\left[g^{t}\right]=0,
$$

$x_{i}^{f}\left[g^{t}\right]$ is also the solution of the boundary value problem (3.21) with $g=\bar{g}^{t}$ and with the boundary conditions:

$$
x_{i^{\prime}}^{f^{\prime}}\left[\bar{g}^{t}\right] \circ I_{i}=\tilde{x}_{i}^{\prime} \circ \gamma_{i}\left[\bar{g}^{t}, \hat{e}\right]=\tilde{x}_{i} \circ \gamma_{i}\left[g^{t}, \hat{e}\right], \quad(i \rightarrow f) .
$$

With this identification, using the $\mathscr{D}_{M}^{i j} \odot \overline{\mathscr{W}}_{M}^{i j}$-invariance of the "classical action" $S\left[g, x_{i}^{f}[g]\right]$, we have

$$
S\left[g^{t}, x_{i}^{f}\left[g^{t}\right]\right]=S\left[\bar{g}^{t}, x_{i^{\prime}}^{f^{\prime}}\left[\bar{g}^{t}\right]\right]=S\left[g_{0}^{t}, x_{i^{\prime}}^{f^{\prime}}\left[g_{0}^{t}\right]\right],
$$

where $g_{0}^{t}$ is the section (3.31) of $\mathscr{B}_{M}^{0}$. form:

The relation between $\tilde{x}_{i}^{\prime}, \tilde{x}_{f}^{\prime}$ and $\tilde{x}_{i}, \tilde{x}_{f}$ in (3.35) can be expressed in the following

$$
\tilde{x}_{i}^{\prime}=\tilde{x}_{i} \circ \tilde{\gamma}_{i}^{t}[\hat{e}], \quad(i \rightarrow f),
$$

where the diffeomorphisms $\tilde{\gamma}_{i}^{t}[\hat{e}], \tilde{\gamma}_{f}^{t}[\hat{e}]$ are uniquely determined by the equations

$$
\begin{gathered}
\text { const } \times \hat{e}=\tilde{\gamma}_{i}^{t *}[\hat{e}]\left(\exp \left(\tilde{\phi}_{i}^{t}\right) \hat{e}\right), \quad(i \rightarrow f), \\
\tilde{\phi}_{i}^{t}=\phi^{t} \circ I_{i} \circ \gamma_{i}^{-1}\left[g^{t}, \hat{e}\right], \quad(i \rightarrow f) .
\end{gathered}
$$

The diffeomorphisms $\tilde{\gamma}_{i}^{t}[\hat{e}], \tilde{\gamma}_{f}^{t}[\hat{e}]$ have the following important feature: they are independent of the choice of decomposition (3.34) and of section $\Xi$ in $\mathscr{B}_{M}$. In fact, for a fixed $\Xi$ various decompositions (3.34) yield the boundary values $\phi_{i}^{t}=\phi^{t} \circ I_{i},(i \rightarrow f)$ which differ by a constant. One can obtain the same effect changing the section $\Xi$ by the action of the group $\overline{\mathscr{W}}_{M}^{i j}$. In both cases the solutions of the equations (3.38) remain unchanged. For sections $\Xi^{\prime}: T_{M}^{R} \ni t \rightarrow g^{\prime t}=f^{t *} g^{t} \in \mathscr{B}_{M}$ the decomposition (3.34) takes the form:

$$
g^{\prime t}=\exp \left(\phi^{t} \circ f^{t}\right) f^{t *} g^{t}
$$


Since

$$
\gamma_{i}\left[f^{*} g, \hat{e}\right]=\gamma_{i}[g, \hat{e}] \circ f \circ I_{i}, \quad(i \rightarrow f)
$$

Eq. (3.39) defines the same function $\tilde{\phi}_{i}^{t}$ as in the case of section $\Xi$,

$$
\tilde{\phi}_{i}^{t}=\phi^{t} \circ f \circ \gamma_{i}^{-1}\left[f^{*} g^{t}, \hat{e}\right]=\phi^{t} \circ \gamma_{i}^{-1}\left[g^{t}, \hat{e}\right], \quad(i \rightarrow f) .
$$

It follows from the definition (3.38) that

$$
\tilde{\gamma}_{i}^{t}\left[\lambda \gamma^{*} \hat{e}\right]=\gamma^{-1} \circ \tilde{\gamma}_{i}^{t}[\hat{e}] \circ \gamma, \quad(i \rightarrow f) .
$$

Therefore the family $\left\{\Gamma_{i}^{t}, \Gamma_{f}^{t}\right\}_{t \in T_{M}^{R}}$ of elements of the group $\mathscr{G}(3.7)$ determined by

$$
\Gamma_{i}^{t}=\Pi_{\mathscr{G}}\left(\hat{e}, \tilde{\gamma}_{i}^{t}[\hat{e}]\right), \quad(i \rightarrow f),
$$

is independent of the choice of $\hat{e}$. Thus it can be considered as an invariant characteristic of the subbundle $\mathscr{B}_{M} \subset \mathfrak{B}_{M}$.

Taking into account the relations (3.36), (3.37), the definitions (3.8), (3.40) and the results $(3.32,33)$, for an arbitrary subbundle $\mathscr{B}_{M} \subset \mathfrak{B}_{M}$ we have

$$
P\left[\bar{c}_{i}, \bar{c}_{f} \mid \mathscr{B}_{M}\right]=\mathrm{const} \int_{0}^{t} d t e^{\pi t}\left[\Pi\left(1-e^{i 2 \pi n t}\right)\right]^{-12} t^{-13} e^{-W\left[\Gamma_{i}^{t} \bar{c}_{i}, \Gamma_{f}^{t} \bar{c}_{f}, t\right]}
$$

In the special cases of subbundles $\mathscr{B}_{M}^{\Gamma}$ for which $\Gamma_{i}^{t}=\Gamma_{i}^{t}=\Gamma$, Eq. (3.41) takes the following simple form:

$$
P\left[\bar{c}_{i}, \bar{c}_{f} \mid \mathscr{B}_{M}^{\Gamma}\right]=P\left[\Gamma \bar{c}_{i}, \Gamma \bar{c}_{f} \mid \mathscr{B}_{M}^{0}\right] .
$$

Let us turn for a moment to the quantum mechanical interpretation of the functionals $P\left[\bar{c}_{i}, \bar{c}_{f} \mid \mathscr{B}_{M}\right]$. A detailed discussion of this point requires a BRST extension of these functionals and the space $\overline{\mathfrak{H}}^{\text {off }}$ with an appropriate inner product. These problems are beyond the scope of this paper and we will restrict ourselves only to some general remarks.

In the gauge $\hat{e}=1$ and in the case of the pointlike contours $\left(\tilde{x}_{i}^{k}=\tilde{x}_{f}^{k}=0, k \neq 0\right)$ the formula (3.32) exactly coincides with the "semi-off-shell" string propagator considered in $[11,17]$. For arbitrary contours, proceeding as in [11], one can rewrite the result (3.32) as

$$
P\left[\tilde{x}_{i}, \tilde{x}_{f} \mid \mathscr{B}_{M}^{0}\right]=\mathrm{const} \int_{0}^{\infty} d t e^{\pi t} \prod_{n>0}\left(1-e^{-2 \pi n t}\right)\left\langle\tilde{x}_{i}\left|e^{-H t}\right| \tilde{x}_{f}\right\rangle,
$$

where $H$ denotes the open string Hamiltonian equal to the zero mode of the density $W(s) \sim P^{2}(s)+x^{\prime 2}(s)$ of quadratic constraints. By introduction of $b-c$ ghost variables and "exponentiation" of the F-P determinant one can construct [18] the BRST extension of the functional (3.43):

$$
P\left[\tilde{x}_{i}, \hat{\Theta}_{i}, \Theta_{i} ; \tilde{x}_{f}, \hat{\Theta}_{f}, \Theta_{f} \mid \mathscr{B}_{M}^{0}\right]=\text { const } \int_{0}^{\infty} d t\left\langle\tilde{x}_{i}, \hat{\Theta}_{i}, \Theta_{i}\left|e^{-\pi t H_{\mathrm{BRST}}}\right| \tilde{x}_{f}, \hat{\Theta}_{f}, \Theta_{f}\right\rangle .
$$

$\widehat{\Theta}_{i}, \widehat{\Theta}_{f}$ and $\Theta_{i}, \Theta_{f}$ denote appropriately defined boundary values of the ghost variables $b$ and $c$, respectively, and $H_{\mathrm{BRST}}$ is a BRST-extended open string 
Hamiltonian. The above form of the BRST-extended string propagator has been also proposed in $[19,20]$.

The formula (3.44) offers a quantum mechanical interpretation of the functional $P\left[\bar{c}_{i}, \bar{c}_{f} \mid \mathscr{B}_{M}^{0}\right]$ (3.32) determined by the subbundle $\mathscr{B}_{M}^{0}$. It can be regarded as the "body" of the integral kernel of the operator inverse to the BRST extended zero mode $W_{0} \sim \int\left(\mathscr{P}^{2}(s)+x^{\prime 2}(s)\right) d s$ of quadratic constraints.

Let us note that since the canonical string Hamiltonian weakly vanishes there is a large amount of freedom in the choice of Hamiltonian governing the evolution in the intrinsic (world sheet) time. In fact one can choose an arbitrary linear combination of constraints even with time dependent coefficients. Roughly speaking the freedom of choice of a subbundle $\mathscr{B}_{M} \subset \mathfrak{B}_{M}$ is related to the freedom of choice of the string Hamiltonian. The interesting question is which subbundles $\mathscr{B}_{M}$ lead to the time independent Hamiltonians, in particular to higher modes of quadratic constraints. The formula (3.42) suggests that it is so for the subbundles $\mathscr{B}_{M}^{\Gamma}$. Such interpretation requires, however, special care since the inner product (3.13) is not $\mathscr{G}$-invariant. In the sequel we will restrict ourselves to the functionals $P\left[\bar{c}_{i}, \bar{c}_{f} \mid \mathscr{B}_{M}\right]$ corresponding to the standard choice of the string Hamiltonian.

Now let us return to the corner conformal anomaly. As it follows from the formula (3.27) the restriction to the space of trajectories $\overline{\mathscr{T}}_{M}^{i j}\left(i, f \mid \mathscr{B}_{M}^{0}\right)$ is insufficient to ensure the decoupling of the conformal factor in the critical dimension. We need a further restriction, which leads to a $\mathscr{D}_{M}^{i j} \odot \overline{\mathscr{W}}_{M}^{i j}$-subbundle of $\mathscr{B}_{M}^{0}$, where $\overline{\mathscr{W}}_{M}^{i j}$ consists of functions $\phi \in \mathscr{W}_{M}^{i j}$ for which

$$
\phi \circ I_{i}=-\phi \circ I_{f}=\text { const . }
$$

In order to describe the space of all such subbundles of $\mathscr{B}_{M}^{0}$ let us fix a "reference" one $-\overline{\mathscr{B}}_{M}^{0}(1)$ generated from the section $\Xi_{0}$ (3.31) of $\mathscr{B}_{M}^{0}$ by the group $\mathscr{D}_{M}^{i j} \odot \overline{\bar{W}}_{M}^{i j}$. Then every subbundle $\overline{\mathscr{B}}_{M}^{0}$ of $\mathscr{B}_{M}^{0}$ can be obtained from $\overline{\mathscr{B}}_{M}^{0}(1)$ by a vertical automorphism of $\mathscr{B}_{M}^{0}$,

$$
\Pi_{\mathscr{B}}^{-1}(t) \ni g \rightarrow e^{\phi^{t}} g \in \Pi_{\mathscr{B}}^{-1}(t) .
$$

It is clear that the function

$$
\chi: T_{M}^{R} \ni t \rightarrow \chi(t)=\exp \left(\phi^{t} \circ I_{i}+\phi^{t} \circ I_{f}\right) \in \mathbb{R}_{+}
$$

provides an invariant characteristic of the subbundle $\overline{\mathscr{B}}_{M}^{0}$.

We will restrict ourselves to the one parameter family of subbundles $\left\{\overline{\mathscr{B}}_{M}^{0}(\ell)\right\}_{l \in \mathbb{R}_{+}}$corresponding to the constant functions $\chi(t) \equiv \ell^{2}$. Note that only for these subbundles the quantum mechanical interpretation of the string propagator given above remains unchanged. For a reason which later becomes clear, we consider the family $\left\{\mathscr{B}_{M}^{0}(\ell)\right\}_{l \in \mathbb{R}_{+}}$of the $\mathscr{D}_{M}^{i j} \odot \mathscr{W}_{M 0^{i j}}^{i j}$ subbundles of $\mathscr{B}_{M}^{0}$ :

$$
\mathscr{B}_{M}^{0}(\ell)=\left\{g \in \mathscr{B}_{M}^{0}, \int_{\Sigma_{i}} e_{i} d s=\int_{\Sigma_{f}} e_{f} d s=\ell\right\}
$$

( $\mathscr{W}_{M 0}^{i j}$ denotes the subgroup of $\mathscr{W}_{M}^{i j}$ of functions vanishing along the $S$-boundary of $M)$. For every $\ell \in \mathbb{R}_{+}$the subbundles $\overline{\mathscr{B}}_{M}^{0}(\ell)$ and $\mathscr{B}_{M}^{0}(\ell)$ lead to the same expression for the propagator. 
We arrive at the following covariant path integral representation of the one parameter family of open string propagators in the space $\mathfrak{H}^{\text {off }}$ :

$$
\begin{aligned}
P\left[\bar{c}_{i}, \bar{c}_{f}, \ell\right] & ={ }_{\bar{J}_{M}^{i j}(i, f \mid \ell)} \mathscr{D}(g, x)\left(\int_{\mathscr{D} i j} \mathscr{D} f\right)^{-1}\left({ }_{W_{M K O}^{i j}} \mathscr{D} \phi\right)^{-1} e^{-S[g, x]} \\
& =\operatorname{const} \times \ell^{5 / 2} \int_{0}^{\infty} e^{\pi t}\left[\prod\left(1-e^{i 2 \pi n t}\right)\right]^{-12} t^{-13} e^{-W\left[\bar{c}_{i}, \bar{c}_{f} \mid t\right]} d t,
\end{aligned}
$$

where the space $\overline{\mathscr{T}}_{M}^{i j}(i, f \mid \ell)$ of the string trajectories consists of $(g, x) \in \mathscr{M}_{M}^{i j} \times \mathscr{E}_{M}^{26}$ fulfilling the condition

$$
\Pi_{\overline{\mathscr{C}}}\left(\hat{e}, x_{i} \circ \gamma_{i}^{-1}[g, \hat{e}]\right)=\bar{c}_{i}, \quad(i \rightarrow f), \quad g \in \mathscr{B}_{M}^{0}(\ell) .
$$

As was mentioned above the string propagator $P\left[\bar{c}_{i}, \bar{c}_{f} \mid \ell\right]$ should be interpreted as the "body" of the integral kernel of some operator in a BRST-extended space $\overline{\mathfrak{H}}^{\text {off }}$. It of course requires some notion of integration in the space $\overline{\mathscr{C}}_{L}^{26}$. But the ultralocal functional measure on $\mathscr{C}_{L}^{26}$ (see (3.13)) is not $\mathbb{R}_{+}$-invariant and cannot be reduced by the F-P method to an ultralocal measure in $\overline{\mathscr{C}}_{L}^{26}$. There are two possible ways to solve this problem.

In the first approach one fixes the $\mathbb{R}_{+}$-invariance in the space $\mathscr{C}_{L}^{26}$, taking the gauge slice $\mathscr{C}_{L}^{26}(\ell) \subset \mathscr{C}_{L}^{26}$ consisting of all metrized contours with fixed intrinsic length $\ell$. On $\mathscr{C}_{L}^{26}(\ell)$ there is the induced ultralocal Riemannian structure which can be used for the construction of the inner product in $\overline{\mathscr{C}}_{L}^{26}$. In the gauge $\overline{\mathscr{S}}_{L}^{\grave{e}}(3.8)$, $\int_{L} \hat{e} d s=\ell$ it is defined by:

$$
\left(\Psi_{e}[\tilde{x}], \Psi_{e}^{\prime}[\tilde{x}]\right)^{l}=\int_{\mathscr{E}_{M L}^{2}} \mathscr{D}^{\hat{e}} \tilde{x}\left(\int_{\hat{W}^{L}} \mathscr{D} \phi\right)^{-1} \bar{\Psi}_{e}[\tilde{x}] \Psi_{e}^{\prime}[\tilde{x}],
$$

where $\mathscr{W}_{L}^{\hat{e}}$ is the subgroup of $\mathscr{W}_{L}$ consisting of the functions fulfilling the condition $\int_{L} \phi \hat{e} d s=0$.

The choice of the gauge slice $\mathscr{C}_{L}^{26}(\ell)$ and of the subbundle $\mathscr{B}_{M}^{0}(\ell)$ which leads to the inner product (3.47) is the third stage of gauge fixing within the formulation based on the space $\overline{\mathfrak{H}}^{\text {off }}$. Hereafter we will call this gauge the fixed length gauge. Note that the resulting formulation does not depend on the choice of $\ell$. Roughly speaking the change of $\ell$ can be compensated by a renormalization of the string wave functionals.

In the second approach, instead of restricting an inner product to $\overline{\mathscr{C}}_{L}^{26}$ one looks for an extension of the string propagator to the space $\mathscr{C}_{L}^{26}$. The extension consistent with the constraint algebra has the form

$$
P\left[c_{i}, c_{f}\right]=\delta\left(\ell_{i}-\ell_{f}\right) P\left[\bar{c}_{i}, \bar{c}_{f} \mid \ell_{i}\right] .
$$

The formula above has been derived from the string propagator in the space $\overline{\mathscr{C}}_{L}^{d}$ and from the fact that the intrinsic string length $\ell$ is a nondynamical variable in the first quantized free string. One would like to have, however, a covariant functional representation of $P\left[c_{i}, c_{f}\right]$ as a sum over string trajectories starting and ending at fixed points in the space $\mathscr{C}_{L}^{26}$. The problem with such a representation is related to the lack of any natural mechanism in the covariant path integral yielding (in the expression for the evolution operator or for the propagator) the delta function for nondynamical variables. 
To see this more explicitly, let us consider the space $\mathscr{T}_{M}^{i j}(i, f)$ of all string trajectories $(g, x) \in \mathscr{M}_{M}^{i j} \times \mathscr{E}_{M}^{26}$ determined by the conditions:

$$
\begin{gathered}
\Pi_{\mathscr{C}}\left(\sigma_{i}^{*} e_{i}, \sigma_{i}^{*} x_{i}\right)=c_{i}, \quad(i \rightarrow f), \\
g \in \mathscr{B}_{M}^{0}\left(\ell_{i}, \ell_{f}\right) \equiv\left\{g \in \mathscr{B}_{M}^{0}: \int_{\Sigma_{i}} e_{i} d s=\ell_{i}, \quad(i \rightarrow f)\right\} .
\end{gathered}
$$

$\left(\mathscr{B}_{M}^{0}\left(\ell_{i}, \ell_{f}\right)\right.$ is the $\mathscr{D}_{M}^{i j} \odot \mathscr{W}_{M 0}^{i j}$-subbundle of $\left.\mathscr{B}_{M}^{0}\right)$.

For every $\ell_{i}, \ell_{f} \in \mathbb{R}_{+}$the functional integral (3.29) over the space $\mathscr{T}_{M}^{i j}(i, f)$ yields the result

$$
\ell_{i}^{-5 / 4} \ell_{f}^{5 / 4} P\left[\bar{c}_{i}, \bar{c}_{f} \mid \ell_{i}\right],
$$

which agrees with (3.48) only for $\ell_{i}=\ell_{f}$. In order to achieve the correct form of the string propagator on the space $\mathscr{C}_{L}^{26}$ one can impose the additional condition for the allowed metrics $g \in \mathscr{B}_{M}^{0}\left(\ell_{i}, \ell_{f}\right)$,

$$
g \in \mathscr{W}_{M 0}^{i j}\left(\mathscr{M}_{M 0}^{i j}\right)
$$

where $\mathscr{M}_{M 0}^{i j}$ consists of the metrics with the zero scalar curvature on $M$. With this condition the space of the allowed metrics is empty for $\ell_{i} \neq \ell_{f}$ and equal to $\mathscr{B}_{M}^{0}(\ell)$ for $\ell_{i}=\ell_{f}=\ell$, which leads to the delta function structure of the propagator. The special choice of the space $\mathscr{T}_{M}^{i j}(i, f)$ of string trajectories determined by (3.49) and (3.50) can be regarded as the third stage of gauge fixing in the formulation based on the space $\mathfrak{H}^{\text {off }}$. We will refer to this choice as to the constant curvature gauge.

\section{The Off-Shell Open String Amplitudes}

The formulae (3.45) and (3.48) provide a well justified (from the geometrical and from the quantum mechanical point of view) starting point for the construction of the off-shell open string amplitudes in the spaces $\overline{\mathfrak{H}}^{\text {off }}$ and $\mathfrak{H}^{\text {off }}$, respectively. The aim of this section is to describe two constructions based on the previous section considerations now generalized to the case of more complicated topologies of the world sheet $M$.

Let us first consider the simplest case of the open string off-shell vertex functional $V\left[\bar{c}_{1}, \bar{c}_{2}, \bar{c}_{3}\right]$ in the space $\overline{\mathscr{C}}_{L}^{26}$. In this case $M$ should be replaced by a hexagon $H$ with distinguished alternating three "timelike" $(T-)$ and three "spacelike" $(S-)$ sides and two involutions $i, j$ of the "timelike" $\left(H_{D}\right)$ and of the "spacelike" $\left(H^{D}\right)$ doublings of $H$, respectively. We define the spaces $\mathscr{M}_{H}^{i j}, \mathscr{E}_{H}^{26}$ and the groups $\mathscr{D}_{H}^{i j}, \mathscr{W}_{H}^{i j}, \overline{\mathscr{W}}_{H}^{i j}, \overline{\mathscr{W}}_{H}^{i j}, \mathscr{W}_{H 0}^{i j}$ by the obvious generalization of conditions used in the case of the rectangle in Sect. 3.

The action of $\mathscr{D}_{H}^{i j} \odot \mathscr{W}_{H}^{i j}$ on $\mathscr{M}_{H}^{i j}$ induces the structure of the principal fibre bundle $[25,26]$ :

$$
\begin{aligned}
\mathscr{D}_{H}^{i j} \odot \mathscr{W}_{H}^{i j} \rightarrow & \mathscr{M}_{H}^{i j} \\
& \downarrow^{\Pi_{T}} \\
& T_{H}^{R}=\mathscr{M}_{H}^{i j} /\left(\mathscr{D}_{H}^{i j} \odot \mathscr{W}_{H}^{i j}\right),
\end{aligned}
$$

where $T_{H}^{R}$ denotes the relative Teichmüller space of hexagon isomorphic to $\mathbb{R}_{+}^{3}$. Note that $T_{H}^{R}$ is isomorphic to the reduced Teichmüller space $T_{H}^{\#}$ [27]. The 
important feature of (4.1) is that there exists a smooth global section with values in the space $\mathscr{M}_{H-1}^{i j}$ of all metrics $g \in \mathscr{M}_{H}^{i j}$ with constant scalar curvature $R(g)=-1$. The remarks above are valid for more complicated topologies of $M$ as well [25-27]. This allows for the straightforward extension of the present considerations to the case of an arbitrary off-shell amplitude.

The space $\mathfrak{B}_{H}$ of all $\mathscr{D}_{H}^{i j} \odot \overline{\mathscr{W}}_{H}^{i j}$-subbundles of (4.1) can be described as in the case of a rectangle. There exists the distinguished subbundle $\mathscr{B}_{H}^{c} \in \mathfrak{B}_{H}$,

$$
\begin{array}{r}
\mathscr{D}_{H}^{i j} \odot \overline{\mathscr{W}}_{H}^{i j} \rightarrow \mathscr{B}_{H}^{c} \\
\left.\right|_{T} ^{\Pi_{T}^{c}} \\
T_{H}^{R}
\end{array}
$$

obtained from the space $\mathscr{M}_{H-1}^{i j}$ by the action of the group $\overline{\mathscr{W}}_{H}^{i j}$. Using $\mathscr{B}_{H}^{c}$ as a "reference" subbundle and repeating the constructions (3.34), (3.38), (3.39), (3.40) of the previous section one can parametrize the space $\mathfrak{B}_{H}$ by the space of functions

$$
T_{H}^{R} \ni t \rightarrow\left(\Gamma_{1}^{t}, \Gamma_{2}^{t}, \Gamma_{3}^{t}\right) \in \mathscr{G}_{L}^{3} .
$$

The generalization of the fixed length gauge (for the case of hexagon) consists in the choice of the one parameter family $\left\{\mathscr{B}_{H}^{c}(\ell)\right\}_{\ell \in \mathbb{R}^{+}}$of $\mathscr{D}_{H}^{i j} \odot \mathscr{W}_{H 0}^{i j}$-subbundles of $\mathscr{B}_{H}^{c}$ :

$$
\mathscr{B}_{H}^{c}(\ell)=\left\{g \in \mathscr{B}_{H}^{c}: \int_{\Sigma_{i}} e_{i} d s=\ell, i=1,2,3\right\} .
$$

In this gauge the space of "trajectories" $\overline{\mathscr{T}}_{H}^{i j}(1,2,3 \mid \ell)$ is defined as the space of all pairs $(g, x) \in \mathscr{M}_{H}^{i j} \times \mathscr{E}_{H}^{26}$ satisfying the conditions:

$$
\Pi_{\overline{\mathscr{G}}}\left(\sigma_{i}^{*} e_{i}, \sigma_{i}^{*} x_{i}\right)=\bar{c}_{i}, \quad i=1,2,3, \quad g \in \mathscr{B}_{H}^{c}(\ell),
$$

where $\sigma_{i}: L \rightarrow \Sigma_{i}, i=1,2,3$ denote arbitrary parametrizations of the components $\Sigma_{i}$ of the $S$-boundary of $H$ and $e_{i}^{2}=I_{i}^{*} g, x_{i}=I_{i}^{*} x, i=1,2,3 .\left(I_{i}: \Sigma_{i} \rightarrow H, i=1,2,3\right.$, denote the inclusions of the components $\Sigma_{i}$ of the $S$-boundary of $H$.)

We define the covariant functional integral representation of the three open strings vertex functional in the fixed length gauge in $\overline{\mathscr{C}}_{L}^{d}$ by:

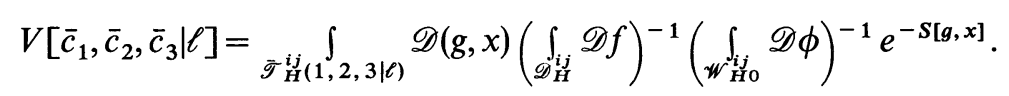

The formal expression above can be consistently evaluated (=defined) by the Faddeev-Popov method. Proceeding along standard lines [28] we obtain:

$$
\begin{aligned}
V\left[\bar{c}_{1}, \bar{c}_{2}, \bar{c}_{3} \mid 1\right]= & \int_{T_{R}^{R}} d^{3} t \times \operatorname{det} M_{g^{t}}\left(\delta \psi_{i} \mid \delta \bar{\chi}_{j}\right)\left(\frac{\operatorname{det}_{A A} P_{g^{t}}^{+} P_{g^{t}}}{\operatorname{det} H\left(P_{g^{t}}^{t}\right)}\right)^{1 / 2} \\
& \times\left(\operatorname{det}_{D N} \mathscr{L}_{g^{t}}\right)^{-13} e^{-S\left[g^{t}, x_{c l}\left[g^{t}\right]\right]},
\end{aligned}
$$

where $T_{H}^{R} \ni t \rightarrow g^{t} \in \mathscr{B}_{H}^{c}(1)$ is a global section of $\mathscr{B}_{H}^{c}(1)$ and $x_{c l}\left[g^{t}\right]$ denotes the solution of the boundary value problem (3.21) generalized to the case of hexagon:

$$
\begin{gathered}
\mathscr{L}_{g^{t}} x_{c l}\left[g^{t}\right]=0, \\
x_{c l}\left[g^{t}\right] \circ I_{i}=\tilde{x}_{i} \circ \gamma_{i}[g, \hat{e}], \quad i=1,2,3, \\
\left(n^{i}\right)^{a} \partial_{a} x_{c l}\left[g^{t}\right]=0 \quad \text { on } \quad \partial M \backslash\left(\Sigma_{1} \cap \Sigma_{2} \cap \Sigma_{3}\right),
\end{gathered}
$$


where $\left(\hat{e}, \tilde{x}_{i}\right) \in \Pi_{\overline{\mathscr{G}}}^{-1}\left(\bar{c}_{i}\right), i=1,2,3$, and the diffeomorphisms $\gamma_{i}[g, \hat{e}]$ are defined by analogy with (3.18).

From the $\mathscr{D}_{H}^{i j} \odot \overline{\mathscr{W}}_{H}^{i j}$-invariance of the integrand in the formula (4.7) it follows that for the decoupling of conformal factor it is enough to consider $\mathscr{D}_{H}^{i j} \odot \overline{\mathscr{W}}_{H^{-}}^{i j}$ subbundles of $\mathscr{B}_{H}^{c}$. As in the case of a rectangle one can fix as a "reference" subbundle the $\mathscr{D}_{H}^{i j} \odot \overline{\bar{W}}_{H}^{i j}$-extension of $\mathscr{B}_{H}^{c}(1): \overline{\mathscr{B}}_{H}^{c}=\overline{\mathscr{W}}_{H}^{i j}\left(\mathscr{B}_{H}^{c}(1)\right)$. Then every $\mathscr{D}_{H}^{i j} \odot \overline{\mathscr{W}}_{H}^{i j}$-subbundle of $\mathscr{B}_{H}^{c}$ can be obtained from $\overline{\mathscr{B}}_{H}^{c}(1)$ by a vertical automorphism of the bundle (4.1):

$$
\Pi_{T}^{-1}(t) \ni g \rightarrow \exp \left(\phi^{t}\right) g \in \Pi_{T}^{-1}(t), \quad \phi^{t} \in \overline{\mathscr{W}}_{H}^{i j},
$$

and is uniquely determined by the function:

$$
\chi_{H}: T_{H}^{R} \ni t \rightarrow \exp \left(\sum_{i=1}^{3} \phi^{t} \circ I_{i}\right) \in \mathbb{R}_{+} .
$$

The $\mathscr{D}_{H}^{i j} \odot \overline{\mathscr{W}}_{H}^{i j}$-subbundle $\overline{\mathscr{B}}_{H}^{c}(\ell)$ corresponding to the constant function $\chi_{H}(t) \equiv \ell^{3}$ yields the same expression for the vertex as $\mathscr{B}_{H}^{c}(\ell)$.

It follows from the corner anomaly [17] that:

$$
V\left[\bar{c}_{1}, \bar{c}_{2}, \bar{c}_{3} \mid \ell\right]=\ell^{15 / 4} V\left[\bar{c}_{1}, \bar{c}_{2}, \bar{c}_{3} \mid 1\right],
$$

therefore the $\ell$-dependence can be compensated by a renormalization of string wave functionals as it was suggested in [17].

Summing up, in the fixed length gauge the "body" of the off-shell formulation of the open bosonic string in the space $\overline{\mathfrak{H}}^{\text {off }}$ includes the inner product $(,)^{1}(3.47)$ in $\overline{\mathfrak{H}}^{\text {off }}$, the off-shell string propagator $P\left[\bar{c}_{i}, \bar{c}_{f} \mid 1\right](3.45)$ and the off-shell three strings vertex functional $V\left[\bar{c}_{1}, \bar{c}_{2}, \bar{c}_{3} \mid 1\right]$ (4.6). The off-shell amplitudes corresponding to more complicated topologies of the world sheet (including handles and holes) can be constructed by a straightforward generalization of the formulae $(4.6,7)$. Let us note that in the gauge (3.10) with $\hat{e} \equiv 1$ on $L$ and for pointlike string states the expression (4.7) exactly coincides with the semi-off-shell vertex discussed in [17] (see also [12] for the closed string case). For the arbitrary contours the formulae (4.7) is the open string version of the off-shell amplitudes considered in $[15,16]$.

Let us now turn to the construction of the off-shell open string amplitudes in the space $\mathfrak{H}^{\text {off }}$. In the case of a hexagon the requirement of the consistency of the Faddeev-Popov method and of the vanishing of the corner anomaly admit a choice of the space of "trajectories" $(g, x) \in \mathscr{M}_{H}^{i j} \times \mathscr{E}_{H}^{26}$ fulfilling the conditions:

$$
\Pi_{\mathscr{C}}\left(\sigma_{i}^{*} e_{i}, \sigma_{i}^{*} x_{i}\right)=c_{i}, \quad i=1,2,3, \quad g \in \mathscr{B}_{H}^{c}\left(\ell_{1}, \ell_{2}, \ell_{3}\right) .
$$

$\mathscr{B}_{H}^{c}\left(\ell_{1}, \ell_{2} \cdot \ell_{3}\right)$ denotes the $\mathscr{D}_{H}^{i j} \odot \mathscr{W}_{H 0^{i j}}^{i j}$ subbundle of $\mathscr{B}_{H}^{c}$ consisting of metrics with the fixed intrinsic lengths:

$$
\ell_{i}=\int_{\Sigma_{i}} e_{i} d s, \quad i=1,2,3
$$

determined by the intrinsic lengths of the contours $c_{1}, c_{2}, c_{3} \in \mathscr{C}_{L}^{26}$.

However, as was discussed in the end of the previous section the analogous choice in the case of a rectangle does not lead to the correct form of the open string propagator and some additional condition for allowed metrics have to be introduced. 
In the case of a hexagon the additional condition takes the form

$$
g \in \mathscr{B}_{H 0}^{-1}=\mathscr{W}_{H 0}^{i j}\left(\mathscr{M}_{H-1}^{i j}\right),
$$

where $\mathscr{B}_{H 0}^{-1}$ is the $\mathscr{D}_{H}^{i j} \odot \mathscr{W}_{H 0^{i j}}^{i j}$ subbundle of (4.2) obtained from the space $\mathscr{M}_{H-1}^{i j}$ by the $\mathscr{W}_{H 0^{i j}}^{i j}$-action. The space $\mathscr{T}_{H}^{i j}(1,2,3) \subset \mathscr{M}_{H}^{i j} \times \mathscr{E}_{H}^{26}$ of "trajectories" in the constant curvature gauge is therefore defined by the conditions:

$$
\Pi_{\mathscr{C}}\left(\sigma_{i}^{*} e_{i}, \sigma_{i}^{*} x_{i}\right)=c_{i}, \quad i=1,2,3, \quad g \in \mathscr{B}_{H}^{c}\left(\ell_{1}, \ell_{2}, \ell_{3}\right) \cap \mathscr{B}_{H 0}^{-1} .
$$

Note that in the case of a hexagon for every $\left(\ell_{1}, \ell_{2}, \ell_{3}\right) \in \mathbb{R}_{+}^{3}$ the intersection $\mathscr{B}_{H}^{c}\left(\ell_{1}, \ell_{2}, \ell_{3}\right) \cap \mathscr{B}_{H 0}^{-1}$ is the fiber of the bundle $\mathscr{B}_{H 0}^{-1}$ over a fixed point in $T_{H}^{R}$. In fact as is well known from the hyperbolic geometry a hexagon with right angles and geodesic sides is completely specified by the lengths of three alternating sides. These lengths (which can be arbitrary positive real numbers) provides the FenchelNielsen coordinates [32] on the relative Teichmüller space $T_{H}^{R}$ of the hexagon $H$,

$$
L_{i}: T_{H}^{R} \ni t \rightarrow L_{i}(t) \in \mathbb{R}_{+} \cdot
$$

As a consequence the final expression for the vertex functional should not include any integration over Teichmüller parameters.

In contrast to the case of a rectangle the construction of an appropriate functional measure on the space $\mathscr{T}_{H}^{i j}(1,2,3)$ is not straightforward. This space is a submanifold of $\mathscr{M}_{H}^{i j} \times \mathscr{E}_{H}^{26}$, and we have the ultralocal $\mathscr{D}_{H}^{i j}$-invariant Riemannian structure determined by (2.3), (2.4) on $\mathscr{M}_{H}^{i j} \times \mathscr{E}_{H}^{26}$. For the functional measure on $\mathscr{T}_{H}^{i j}(1,2,3)$ related to the induced metric even in the critical dimension the conformal anomaly does not vanish. In order to overcome this problem we introduce the larger space $\tilde{T}_{H}^{i j}(1,2,3)$ defined by the conditions

$$
\Pi_{\mathscr{\mathscr { G }}}\left(\sigma_{i}^{*} e_{i}, \sigma_{i}^{*} x_{i}\right)=\Pi\left(c_{i}\right), \quad i=1,2,3, \quad g \in \mathscr{B}_{H 0}^{-1},
$$

where $\Pi: \mathscr{C}_{L}^{26} \rightarrow \overline{\mathscr{C}}_{L}^{26}$ denotes the canonical projection in the bundle $\mathscr{C}_{L}^{26} \rightarrow \mathscr{C}_{L}^{26} / \mathbb{R}_{+}$. In addition we introduce the delta function on $T_{H}^{R}$,

$$
\prod_{i=1}^{3} \delta\left(\ell_{i}-L_{i}(t)\right)
$$

where $L_{i}(t)$ are the Fenchel-Nielsen coordinates (4.11) on $T_{H}^{R}$. After the substitution $t=\Pi_{T}(g)$ this delta function can be regarded as a $\mathscr{D}_{H}^{i j} \odot \mathscr{W}_{H 0}^{i}$-invariant "characteristic" functional of the submanifold $\mathscr{T}_{H}^{i j}(1,2,3) \subset \widetilde{\mathscr{T}}_{H}^{i j}(1,2,3)$.

In the constant curvature gauge we define the functional integral representation of the three open strings vertex functional $V\left[c_{1}, c_{2}, c_{3}\right]$ in the space $\mathscr{C}_{L}^{26}$ by:

$$
V\left[c_{1}, c_{2}, c_{3}\right]=\underset{\tilde{T}_{H}^{i,(1,2,3)}}{\int} \mathscr{D}(g, x)\left(\int_{\mathscr{O}_{H} i} \mathscr{D} f\right)^{-1}\left(\int_{\mathscr{W}_{H i}^{i j}} \mathscr{D} \phi\right)^{-1} \prod_{i=1}^{3} \delta\left(\ell_{i}-L_{i}[g]\right) e^{-S[g, x]}
$$

The path integral above fulfills the requirement of the consistency of the Faddeev-Popov method and can be evaluated (=defined) in the critical dimension:

$$
\begin{gathered}
V\left[c_{1}, c_{2}, c_{3}\right]=\int_{T R} d^{3} t \prod_{i=1}^{3} \delta\left(\ell_{i}-L_{i}(t)\right) \\
\times \operatorname{det} M_{g^{t}}\left(\delta \psi_{i} \mid \delta \bar{\chi}_{j}\right)\left(\frac{\operatorname{det}_{A A} P_{g^{t}}^{+} P_{g^{t}}}{\operatorname{det} H\left(P_{g^{t}}^{+}\right)}\right)^{1 / 2}\left(\operatorname{det}_{D N} \mathscr{L}_{g^{t}}\right)^{-13} e^{-S\left[g^{t}, x_{c l}\left[g^{t}\right]\right]}
\end{gathered}
$$

(all symbols are defined as in the formula (2.24)). 
One can simplify the expression (4.15) using the Fenchel-Nielsen coordinates on $T_{H}^{R}$. In these coordinates the Weil-Petersson volume element on $T_{H}^{R}$ has the especially simple form: $d \omega^{W P}=d L_{1} d L_{2} d L_{3}$ [32]. Taking into account the relation:

$$
d \omega^{W P}=d^{3} t \frac{\operatorname{det} M_{g^{t}}\left(\delta \psi_{i} \mid \delta \bar{\chi}_{j}\right)}{\operatorname{det} H\left(P_{g^{t}}^{+}\right)}
$$

we arrive at the following expression for the vertex:

$$
V\left[c_{1}, c_{2}, c_{3}\right]=\left(\operatorname{det}_{A A} P_{g^{t}}^{+} P_{g^{t}}\right)^{1 / 2}\left(\operatorname{det}_{D N} \mathscr{L}_{g^{t}}\right)^{-13} e^{-S\left[g^{t}, x_{c l}\left[g^{t}\right]\right]} .
$$

Note that the right-hand side of (4.16) is $\mathscr{D}_{H}^{i j}$-invariant but not $\mathscr{W}_{H 0}^{i j}$-invariant and must be calculated for the metrics $g^{t}$ with constant scalar curvature $R=-1$ and with the boundary conditions (4.9).

The generalization for more complicated topologies of the world sheet $M$ is now straightforward. The off-shell open string amplitudes have the following general structure:

$$
V\left[c_{i}\right]_{i=1, \ldots, n}=\int_{\hat{T}_{R}} d \hat{\omega}^{W P}\left(\operatorname{det}_{A A} P_{g^{t}}^{+} P_{g^{t}}\right)^{1 / 2}\left(\operatorname{det}_{D N} \mathscr{L}_{g^{t}}\right)^{-13} e^{S\left[g^{t}, x_{c l}\left[g^{t}\right]\right]} .
$$

$\widehat{T}_{M}^{R}$ denotes the subspace of $T_{M}^{R}$ defined by the equations $L_{i}=\ell_{i}(i=1, \ldots, n)$ where $L_{i}$ are the Fenchel-Nielsen coordinates corresponding to the "spacelike" segments of the boundary of $M$, and $d \hat{\omega}^{W P}$ is the volume element on $T_{M}^{R}$ obtained from $d \omega^{W P}$ by omitting the factor $d L_{1}, \ldots, d L_{n}$. The integrand in (4.17) is evaluated for any section $T_{M}^{R} \ni t \rightarrow g^{t} \in \mathscr{M}_{M}^{i j}$ with values in $\mathscr{M}_{M-1}^{i j}$ and fulfilling the obvious generalization of the boundary conditions (4.9).

The simple form of (4.16) and (4.17) is a consequence of the choice of functional measure on $\mathscr{T}_{H}^{i j}(1,2,3)$ or more precisely of the "characteristic" functional (4.13). It should be clarified that it is not completely determined by the requirement of vanishing of the conformal anomaly. Our choice is motivated by the special role of the Fenchel-Nielsen coordinates in the construction of Riemann surfaces from simpler building blocks [32]. The idea to extend this construction to multiloop closed string amplitudes has been proposed by D'Hoker and Phong [28].

Summing up, in the constant curvature gauge the "body" of the off-shell formulation of the open bosonic string in the space $\mathfrak{H}^{\text {off }}$ includes the inner product (3.13), the off-shell string propagator (3.48) and the family of the off-shell amplitudes (4.17). Constructing these amplitudes in the space $\mathfrak{H}^{\text {off }}$ is equivalent to extending the corresponding objects defined on the space $\overline{\mathscr{C}}_{L}^{26}$. As was mentioned above the path integral techniques are insufficient for this purpose and should be supplemented by some additional constructions $(4.10,12-14)$. It should be stressed that the extension presented above is based on purely geometrical arguments. Its quantum mechanical justification requires a BRST-extension of the present formalism.

\section{Conclusions}

\subsection{The Open String Quantum Mechanics} in the Position Representation

The considerations of Sect. 3 provide the first step of the covariant functional quantization of the open bosonic string in the position representation. It was 
shown that the general scheme described in Sect. 3.1 leads to the space $\overline{\mathfrak{H}}^{\text {off }}$ of the string wave functionals defined in the space of contours $\overline{\mathscr{C}}_{L}^{26}$ with the inner product $(,)^{1}$ (3.47). The physical string states are described by the $\mathscr{G}$-invariant wave functionals fulfilling the on mass shell condition uncoded in the propagator $P\left[\bar{c}_{i}, \bar{c}_{f} \mid 1\right]$ (3.45). There exists another formulation based on the space $\mathfrak{H}^{\text {off }}$ of the string wave functionals defined on $\mathscr{C}_{L}^{26}$ with the inner product $($,$) (3.13). In this$ case the $\mathscr{G}$-invariance should be replaced by the $\mathscr{G} \times \mathbb{R}_{+}$-invariance and the propagator $P\left[c_{i}, c_{f}\right]$ takes the form (3.48).

In both formulations the string propagator is not invertible. This is the basic reason for constructing a BRST extension. The part of this construction concerning the BRST extended propagator $P\left[\bar{c}_{i}, \bar{c}_{f} \mid 1\right]$ in the fixed length gauge and in the gauge (3.10) with $\hat{e}=1$ on $L$ is rather well understood $[18,19]$. Let us note however that a consistent geometrical setting of a BRST formulation including a BRST-extension of the inner product and a cohomological description of the residual gauge invariance is still an open problem and requires a better insight into the geometry of BRST-invariant functional measures.

In order to complete the covariant first functional quantization described in Sect. 3, besides the BRST extension, one should find the whole space of the wave string functionals corresponding to the particle on-shell open string states. These wave functionals are determined as $\mathscr{G}_{L}$-invariant eigenfunctions of the operators of the momenta and the angular momenta, fulfilling the on-mass shell condition. One can expect that a functional integral over surfaces diffeomorphic to the half-disc with a vertex operator attached to the "timelike" boundary provides a solution to this problem [34].

The general structure of the gauge fixing used in Sect. 3 is described in Table 1.

The first stage consisting in the choice of a model manifold $M$ reduces the large space of gauge transformations of the BDHP action to the group $\mathscr{D}_{M} \odot \mathscr{W}_{M}$. It is further restricted to the group $\mathscr{D}_{M}^{i j} \odot \mathscr{W}_{M}^{i j}$ by the requirement of the consistency of the F-P method. It also follows from this requirement that the space of boundary conditions for string trajectories should be defined in a $\mathscr{D}_{M}^{i j}$-invariant way. The simplest admissible choices are therefore $\mathscr{K}_{L}^{26}, \overline{\mathscr{C}}_{L}^{26}, \mathscr{C}_{L}^{26}$. But as it was discussed in the introduction the choice of $\mathscr{K}_{L}^{26}$ leads to the path integral (1.3) in which an

Table 1. (See text)

\begin{tabular}{llll}
\hline & & $\begin{array}{l}\text { Space of boundary } \\
\text { conditions }\end{array}$ & $\begin{array}{l}\text { Space of string } \\
\text { trajectories }\end{array}$ \\
\hline I & $\begin{array}{l}\mathfrak{B}^{\text {off }} \\
\mathfrak{B}^{\text {off }}\end{array}$ & $L$-fixed & $M$-fixed \\
II & $\bar{B}^{\text {off }}$ & $(3.10)$ & $(3.25)$ \\
& & conformal gauges & $\begin{array}{l}\text { conformal gauges } \\
(3.25)\end{array}$ \\
& $\mathfrak{B}^{\text {off }}$ & $(3.5)$ & $\begin{array}{l}\text { fixed length gauge } \\
\text { III }\end{array}$ \\
$\overline{\mathfrak{B}}^{\text {off }}$ & $\mathscr{G}_{L}, \mathscr{C}_{L}^{26}(\ell)$ & $\begin{array}{l}\text { constant curvature } \\
\text { gauge }\end{array}$ \\
& $\mathfrak{B}^{\text {off }}$ & $\mathscr{G}_{L} \times \mathbb{R}_{+}$ & \\
\hline
\end{tabular}


averaging over boundary reparametrizations appears. On the other hand the comparison with the canonical quantization suggests that the path integral (1.1) with a suitable $x$-dependent gauge should yield the light-cone string propagator. The simplest choices admitting the explicit covariant (with respect to the symmetry of the target space) formulation are therefore $\overline{\mathscr{C}}_{L}^{26}, \mathscr{C}_{L}^{26}$.

The requirement of explicit covariance imposes some restrictions on the possible choice of gauges at the second stage - they cannot involve conditions for the $x$-variables. In the case of $\overline{\mathscr{C}}_{L}^{26}$ every $\tilde{x}$-independent gauge is a 1-dim conformal gauge (3.10). In the case of $\mathscr{C}_{L}^{26}$ there exist more general gauges but they lead to an inessential generalization of the formulation based on the 1-dim conformal ones (3.5). The 2-dimensional conformal gauges (3.25) form a special class of $x$-independent gauges in the space of trajectories. In these gauges the mechanism of the decoupling of the conformal factor in the critical dimension is rather simple. Since there is no $\mathscr{D}_{M}^{i j}$-anomaly one can expect that more general $x$-independent gauges should lead to the same functionals $P\left[\bar{c}_{i}, \bar{c}_{f} \mid 1\right], P\left[c_{i}, c_{f}\right]$.

The third stage of the gauge fixing has a quite different nature. It consists in the choice of the form of the conditions determining the subspace of the physical offshell states. In our case these conditions are the $\mathscr{G}_{-}\left(\mathscr{G} \times \mathbb{R}_{+}-\right)$invariance of the wave functionals defined on the space $\overline{\mathscr{C}}_{L}^{26}\left(\mathscr{C}_{L}^{26}\right.$, respectively). According to the considerations of Subsects. 3.1, 3.2 the space of off-shell physical states $\widetilde{\mathfrak{H}}_{p h}^{\text {off }}=\mathfrak{H}_{p h}^{\text {off }}$ has the quantum mechanical interpretation of the space of all string states annihilated by every constraint linear in momenta. In the canonical analysis of a system with first class constraints one can determine the same constraint surface by various choices of a set of constraint functions. This freedom corresponds to the freedom of choice of conditions determining the space $\overline{\mathfrak{H}}_{p h}^{\text {off }}\left(\mathfrak{H}_{p h}^{\text {off }}\right)$. Note that our choice is related to the standard choice of the subalgebra of linear constraints $\left(V(\sigma) \sim \mathscr{P}(\sigma) x^{\prime}(\sigma)\right)$ in string theory.

The second part of the third stage of the scheme presented in Table 1 plays a similar role. It fixes the freedom of choice of space of allowed string trajectories. As was argued in Subsect. 3.3 the fixed length gauge in $\overline{\mathfrak{H}}^{\text {off }}$ and the constant curvature one in $\mathfrak{H}^{\text {off }}$ are related to the standard choice of a string Hamiltonian. It is an interesting problem to find a BRST extension and a quantum mechanical interpretation of the functionals (3.41) corresponding to arbitrary choices of $\overline{\mathscr{D}}_{M}^{i j} \odot \overline{\mathscr{W}}_{M}^{i j}-\left(\mathscr{D}_{M}^{i j} \odot \mathscr{W}_{M 0^{-}}^{i j}\right)$ subbundles of $\mathscr{M}_{M}^{i j}$. The third stage of gauge fixing is therefore related to the choice of set of constraint operators determining the space of the physical on-mass shell string states.

Finally let us note that the fixed length gauge has the 1-dim part consisting in the choice of gauge slice $\mathscr{C}_{L}^{26}(\ell) \in \mathscr{C}_{L}^{26}$ which fixes the choice of inner product in the space $\overline{\mathfrak{H}}^{\text {off }}$.

\subsection{Sewing of the Off-Shell Open String Amplitudes}

The potential usefulness of the covariant off-shell formulation of string theory is related to the hope that within this formalism one can correctly describe the Feynman rules of the interacting string field theory. The central problem of this approach is to find the sewing rules for amplitudes. In the case of the closed bosonic string the important progress in this direction has been recently made $[15,16]$. 


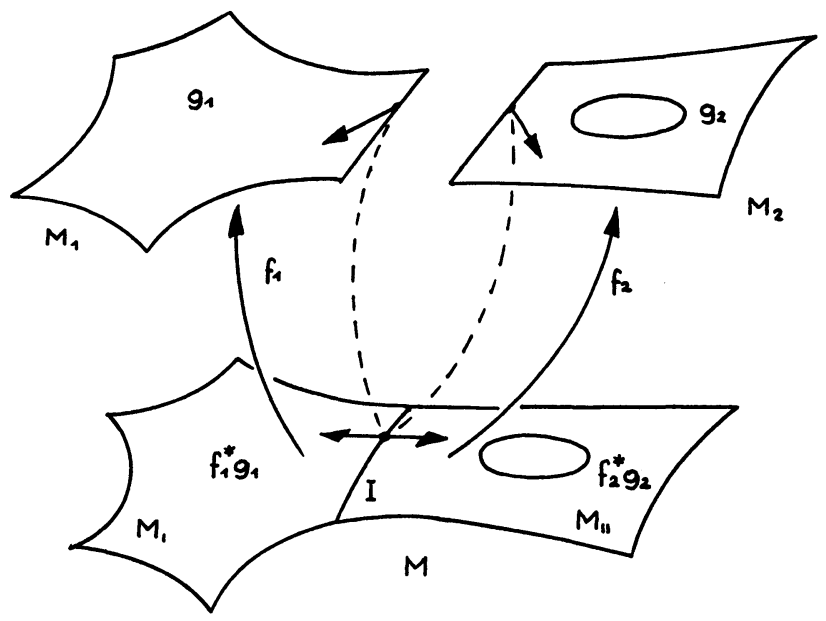

The considerations of the present paper provides the geometrical and quantum mechanical setting for the "body" of the off-shell formulation in $\overline{\mathfrak{H}}^{\text {off }}$ and $\mathfrak{H}^{\text {off }}$. The detailed discussion of the sewing amplitudes requires the BRST extension, so we will restrict ourselves only to a few remarks concerning these elements of the sewing problem which crucialy depends on the "body" of our formalism.

In the formulation based on the space $\overline{\mathfrak{H}}^{\text {off }}$ the off-shell amplitude for the surface $M$ has the structure of a finite dimensional integral $(4,7)$ over the moduli space $m_{M}$ of $M$. The BRST-extended integrand of this expression can be regarded as a partition function on $M$ depending on the boundary values of the ghosts and of the $x$-variables. With our choice of boundary conditions for trajectories (4.5) this partition function is a $\mathscr{D}_{M}^{i j} \odot \mathscr{W}_{M 0}^{i j}$-invariant functional on metrics on $M$. As it was pointed out in [15] the sewing problem consists of two elements: the sewing of partition functions and the sewing of integrals over moduli spaces.

The sewing property of partition functions crucially depends on the third stage of gauge fixing which consists in the choice of the $\mathscr{D}_{M}^{i j} \odot \mathscr{W}_{M 0}^{i j}$-subbundle $\mathscr{B}_{M}^{*}$ of allowed metrics for each topological type of $M$. In the fixed length gauge $\mathscr{B}_{M}^{*}=\mathscr{B}_{M}^{c}(1)$, where $\mathscr{B}_{M}^{c}(1)$ is defined by the obvious generalization of the formula (4.4). The important feature of this gauge is that it possesses the following sewing property: for arbitrary topological types of $M_{1}$ and of $M_{2}$ and for every $t_{1} \in T_{M_{1}}^{R}$, $t_{2} \in T_{M_{2}}^{R}$ such that $L_{I}\left(t_{1}\right)=L_{I}\left(t_{2}\right)$, there exist metrics $g_{1} \in \mathscr{B}_{M_{1}}^{*}, g_{2} \in \mathscr{B}_{M_{1}}^{*}$ over $t_{1}$ and $t_{2}$, respectively, which can be glued together into a smooth metric $g \in \mathscr{B}_{M}^{*}$. The details of this glueing procedure are presented in Fig. 2.

By an appropriate choice of diffeomorphisms $f_{1}: M_{I} \rightarrow M_{1}, f_{2}: M_{I I} \rightarrow M_{2}$ one can always achieve the coincidence of the normal directions of the metrics $f_{1}^{*} g_{1}$, $f_{2}^{*} g_{2}$ along the submanifold $I=M_{I} \cap M_{I I} \subset M$. Then changing the metric $g_{1}$ (or $g_{2}$ ) along the fibre of $\mathscr{B}_{M 1}^{*}$ (or of $\mathscr{B}_{M 2}^{*}$ ) one can also achieve the coincidence of the induced einbeins on $I$ and of other metric components in such a way that $f_{1}^{*} g_{1}$ and $f_{2}^{*} g_{2}$ form a smooth metric $g$ on $M$. All metrics $g$ on $M$ obtained in this way are $\mathscr{D}_{M}^{i j} \odot \mathscr{W}_{M 0}^{i j}$-equivalent. The sewing property of the gauge fixing means that these metrics belong to the subbundle $\mathscr{B}_{M}^{*}$. 
The partition function considered as a functional of $(M, g)$ is invariant with respect to the transformations $(M, g) \rightarrow\left(f^{-1} M, f^{*} g\right)$ generated by arbitrary diffeomorphisms $f: M^{\prime} \rightarrow M$. Therefore in every gauge with the sewing property the sewing problem for partition functions can be reduced to the one of sewing at a fixed conformal structure which was recently solved [16]. Moreover, if we use the fixed length gauge the sewing of the partition functions for the $x$ variables is described by the path integral over $\overline{\mathscr{C}}_{L}^{d}$ used in the definition of the inner product $(,)^{1}$ (3.47). The interesting question is how many gauges with the sewing property, consistent with the inner product $(,)^{1}$ do exist.

It is worthwhile to compare at this point the formulation presented in this paper with that proposed in $[15,16]$. In the fixed length gauge and in the gauge (3.10) in $\mathscr{C}_{L}^{26}$ the off-shell amplitudes constructed in Sect. 3 coincide with the functionals considered in [15] (suitably modified to the open string case). The difference in both approaches consists in different geometrical interpretations of the objects involved. In [15] off-shell amplitudes are considered as functionals of parametrized contours. In such a formulation there are no relations between a conformal structure on a surface $M$ and parametrizations of the $S$-boundaries of $M$. Note that such a relation is necessary for sewing rules for partition functions. In our formulation it is determined by the choice of the space of allowed "trajectories" in the path integral representation of an off-shell amplitude. It will be interesting to interpret within this framework a special relation constructed in [15] by means of holomorphic quadratic differentials on $M$.

Let us now briefly comment on the second element of the sewing problem. The moduli spaces $m_{M_{1}}, m_{M_{2}}$ of surfaces $M_{1}, M_{2}$ include the parameters related to the lengths of the $S$-boundary segments $\Sigma_{i}$ along which $M_{1}$ and $M_{2}$ are sewn. In the case of the open string we have therefore one redundant parameter for each $\Sigma_{i}$. As was argued in [15] these redundant parameters can be removed by the insertion of the inverse of the off-shell string propagator "between" the sewn amplitudes. It does not solve, however, the sewing problem completely. In general the resulting expression involves as infinite overcounting of the moduli space [15].

The formulation in the space $\mathfrak{H}^{\text {off }}$ provides a new alternative approach to the interacting open string. The remarks above concerning the sewing rules for partition functions apply to this case as well. The constant curvature gauge has a similar sewing property and there appears the question of the uniqueness of this gauge. In contrast to the previous approach the off-shell amplitudes do not contain any integration over moduli parameters related to the lengths of $S$-boundary segments. It leads to a simplification of the sewing rules for amplitudes. They can be sewn immediately by means of a BRST-extended inner product in $\mathscr{C}_{L}^{26}$ which provides (with the correct measure!) the missing integration over lengths of common boundary segments. Note that the propagator is not present in this sewing procedure. The only role it plays is the determination of the wave functionals corresponding to on-shell string states. It should be stressed that the sewing in the space $\mathfrak{H}^{\text {off }}$ leads to exactly the same overcounting as in the formulation based on the space $\overline{\mathfrak{H}}^{\text {off }}$. The sewing procedure in both formulations should be therefore supplemented by suitable rules ensuring a single cover of the moduli space. Whether such rules exist is still an open question. 
Acknowledgements. I am very grateful to S. Carlip for his critical remarks about the first version of this paper and for acquainting me with his unpublished preprint [15, third reference]. I would like to thank M. Klimek, Z. Hasiewicz, and J. Sobczyk for helpful discussions and the Commun. Math. Phys. referee for calling my attention to [26, 27].

\section{References}

1. Polyakov, A.M.: Phys. Lett. 103B, 207, 211 (1981)

2. Durhuus, B., Nielsen, H.B., Olesen, P., Petersen, J.L.: Nucl. Phys. B 196, 498 (1982) Durhuus, B., Olesen, P., Petersen, J.L.: Nucl. Phys. B 198, 157 (1982); B 201, 176 (1982)

3. Alvarez, O.: Nucl. Phys. B 216, 125 (1983)

4. D'Hoker, E., Phong, D.H.: Nucl. Phys. B 269, 205 (1986)

5. Gilbert, G.: Nucl. Phys. B 277, 102 (1986)

Polchinski, J.: Commun. Math. Phys. 104, 37 (1986)

6. Moore, G., Nelson, P.: Nucl. Phys. B 266, 58 (1986)

7. Weinberg, S.: Phys. Lett. 156B, 309 (1985)

8. Belavin, A.A., Knizhnik, V.G.: Phys. Lett. 168B, 201 (1986)

Catenacci, R., Cornalba, M., Martellini, M., Reina, C.: Phys. Lett. 172 B, 328 (1986)

Bost, J.B., Jolicoeur, T.: Phys. Lett. 174B, 273 (1986)

9. Gava, E., Iengo, R., Jayaraman, T., Ramachandran, R.: Phys. Lett. 168B, 207 (1986)

Namazie, M.A., Rajeev, S.: Nucl. Phys. B 277, 332 (1986)

Manin, Yu.I.: Phys. Lett. 172B, 184 (1986)

Belavin, A.A., Knizhnik, V.G.: Sov. Phys. JETP 64, 214 (1986)

10. Rodrigues, J.P.: Phys. Lett. 178B, 350 (1986); 202 B, 227 (1988); J. Math. Phys. 28, 2669 (1987) Burgess, C.P., Morris, T.R.: Nucl. Phys. B 256, 256 (1987)

11. Cohen, A., Moore, G., Nelson, P., Polchinski, J.: Nucl. Phys. B 267, 143 (1986)

12. Cohen, A., Moore, G., Nelson, P., Polchinski, J.: Proc. Workshop on Unified String Theory. Green, M., Gross, D. (eds.). Singapore: World Scientific 1986; Nucl. Phys. B 281, 127 (1986)

13. Blau, S.K., Clements, M.: Nucl. Phys. B 284, 118 (1987)

Blau, S.K., Clements, M., McClain, B.: Univ. of Texas preprint UTTG-29-86 (1986)

14. Blau, S.K., Carlip, S., Clements, M., DellaPietra, S., DellaPietra, V.: Nucl. Phys. B 301, 285 (1988)

15. Carlip, S.: Phys. Lett. 209B, 464 (1988); Bordered surfaces, off-shell amplitudes, sewing, and string theory. Lectures given at XXV Winter School of Theoretical Physics, to appear in Proceedings, Birkhäuser, Basel, Boston 1989; "What We Think We Know About Sewing," unpublished

16. Carlip, S., Clements, M., DellaPietra, S., DellaPietra, V.: Inst. of Advanced Study preprint IASSNS/HEP-88/53

17. Varughese, C., Weisberger, W.I.: Phys. Rev. D 37, 981 (1988)

18. Birmingham, D., Torre, C.G.: Phys. Lett. 205B, 289 (1988)

19. Lee, T.: Ann. Phys. 183, 191 (1988)

20. Karchev, N.I.: Phys. Lett. B 212, 158 (1988)

21. Redlich, A.N.: Phys. Lett. 205B, 295 (1988); Inst. of Advanced Study preprint IASSNS/HEP-87/49 (1987)

22. Ohrndorf, T.: Heidelberg preprint HD-THEP-87-12 (1987)

23. Feynman, R.P., Hibbs, A.R.: Quantum mechanics and path integrals. New York: MacGrawHill 1965

24. Brink, L., Di Vecchia, P., Howe, P.: Nucl. Phys. B 118, 76 (1977)

25. Earle, C.J.: Trans. Am. Math. Soc. 126, 54 (1967)

Earle, C.J., Schatz, A.: J. Diff. Geom. 4, 169 (1970)

26. Earle, C.J.: Moduli of surfaces with symmetries. In: Advances in the theory of Riemann surfaces, 1969 Stony Brook Conference. Ahlfors, L. (ed.). Princeton, NJ: Princeton University Press 1971 
